\title{
A Note on Cube-Full Numbers in Arithmetic Progression
}

\author{
Mingxuan Zhong $\mathbb{D}^{1}$ and Yuankui Ma $\mathbb{D}^{2}$ \\ ${ }^{1}$ School of Mathematics and Statistics, Shaanxi Normal University, Xi'an 710119, Shaanxi, China \\ ${ }^{2}$ School of Science, Xi'an Technological University, Xi'an 710021, Shaanxi, China
}

Correspondence should be addressed to Yuankui Ma; mayuankui@xatu.edu.cn

Received 21 January 2021; Accepted 16 February 2021; Published 10 March 2021

Academic Editor: Tingting Wang

Copyright (c) 2021 Mingxuan Zhong and Yuankui Ma. This is an open access article distributed under the Creative Commons Attribution License, which permits unrestricted use, distribution, and reproduction in any medium, provided the original work is properly cited.

We obtain an asymptotic formula for the cube-full numbers in an arithmetic progression $n \equiv l(\bmod q)$, where $(q, l)=1$. By extending the construction derived from Dirichlet's hyperbola method and relying on Kloosterman-type exponential sum method, we improve the very recent error term with $x^{(118 / 4029)}<q$.

\section{Introduction and Main Results}

Let $k>1$ be a fixed integer and $n$ be a positive integer. We call $n$ a powerful number (or $k$-full number) if $n=1$ or for a prime $p$ dividing $n, p^{k}$ also divides $n$. Let $\mathscr{P}_{k}$ denote the set of powerful numbers. Suppose $k=2,3$, and this defines square-full numbers and cube-full numbers, respectively. Erdös and Szerkeres [1] first introduced powerful numbers and gave

$$
\sum_{n \leq x, n \in \mathscr{P}_{k}} 1=\sum_{m=k}^{2 k-1} c_{k, m} x^{(1 / m)}+\Delta_{k}(x)
$$

where $c_{k, m}$ are effective constants and $\Delta_{k}(x) \ll x^{(1 /(k+1))}$. From then on, many authors have studied the powerful numbers and got a lot of relevant conclusions (see [2-18] and references therein).

In 2013, Liu and Zhang [19] investigated the distribution of square-full numbers in arithmetic progressions and got an asymptotic formula

$$
\sum_{\substack{n \leq x, n \in \mathscr{P}_{2} \\ n \equiv l(\bmod q)}} 1=\alpha(l, q) x^{(1 / 2)}+O\left(q^{(49 / 141)+\varepsilon} x^{(19 / 47)+\varepsilon}\right),
$$

under the condition of $(q, l)=1$. By utilizing the method of exponent pairs, Srichan [20] then obtained

$$
\begin{aligned}
& \sum_{\substack{n \leq x, n \in \mathscr{P}_{2} \\
n \equiv l(\bmod q)}} 1=\sum_{k=2}^{3} \beta_{k}(\ell, q) x^{(1 / k)}+O\left(q^{(3 / 2)+\varepsilon} x^{(1 / 6)}\right), \\
& \sum_{\substack{n \leq x, n \in \mathscr{P}_{3} \\
n \equiv l(\bmod q)}} 1=\sum_{k=3}^{5} \gamma_{k}(\ell, q) x^{(1 / k)}+O\left(q^{(127 / 92)+\varepsilon} x^{(7 / 46)}\right),
\end{aligned}
$$

where the error terms had been corrected by Watt [MR3265055].

Recently, Chan [21] got a new asymptotic formula

$$
\sum_{\substack{n \leq x, n \in \mathscr{P}_{2} \\ n \equiv l(\bmod q)}} 1=\sum_{k=2}^{3} \delta_{k}(\ell, q) x^{(1 / k)}+O_{\varepsilon}\left(\left(x^{(1 / 6)} q^{(1 / 12)}+\frac{x^{(1 / 5)}}{q^{(1 / 5)}}\right) q^{\varepsilon}\right)
$$

which improved his own result with Tsang [22]. As a critical step, he [21] mainly dealt with a sum in the form of by following closely Montgomery and Vaughan's construction [23]. It is somewhat similar to Dirichlet's hyperbola method shown in Figure 1.

$$
\sum_{\substack{a^{2} b^{3} \leq x \\ a^{2} b^{3} \equiv l(\bmod q)}} 1
$$




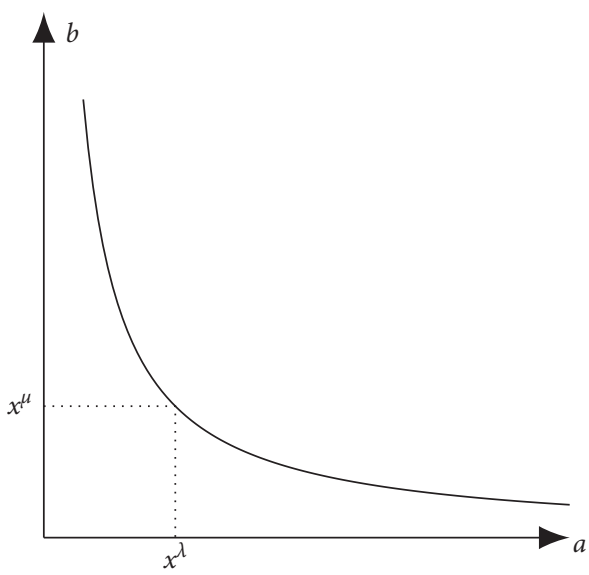

FIgURE 1: Dirichlet's hyperbola method.

Actually, they divided the above sum into four parts as shown in Figure 2 and then discussed them separately.

Motivated by this idea, we turn to discuss the following sum with three parameters:

$$
\sum_{\substack{a^{3} b^{4} c^{5} \leq x \\ a^{3} b^{4} c^{5} \equiv l(\bmod q)}} 1 .
$$

By extending the construction from Montgomery and Vaughan [23], the summation (5) is divided into eight parts as shown in Figure 3.

Then, relying again on Kloosterman-type exponential sum method, an asymptotic formula of (5) is obtained. Finally, an asymptotic formula of cube-full numbers in an arithmetic progression is derived.

Before we formulate our result we need to give some definitions that will be used below. For modulus $q$, we define

$$
\begin{aligned}
& N_{3}(n ; q):=\#\left\{x(\bmod q): x^{3} \equiv n(\bmod q),(x, q)=1\right\}, \\
& N_{4}(n ; q):=\#\left\{x(\bmod q): x^{4} \equiv n(\bmod q),(x, q)=1\right\}, \\
& N_{5}(n ; q):=\#\left\{x(\bmod q): x^{5} \equiv n(\bmod q),(x, q)=1\right\} .
\end{aligned}
$$

It is clear that $N_{3}\left(n a^{3} ; q\right)=N_{3}(n ; q)$, $N_{4}\left(n a^{4} ; q\right)=N_{4}(n ; q)$, and $N_{5}\left(n a^{5} ; q\right)=N_{5}(n ; q)$ for any $(a, q)=1$, and $\quad N_{3}(n ; q)=N_{4}(n ; q)=N_{5}(n ; q)=0 \quad$ if $(n, q)>1$.

Theorem 1. For $(l, q)=1$,

$$
\begin{aligned}
\sum_{\substack{n \leq x, n \in \mathscr{P}_{3} \\
n \equiv l(\bmod q)}} 1= & A_{q}(l) \frac{x^{(1 / 3)}}{q}+B_{q}(l) \frac{x^{(1 / 4)}}{q}+C_{q}(l) \frac{x^{(1 / 5)}}{q} \\
& +O\left(\left(x^{(49 / 360)} q^{(1 / 2)}+\frac{x^{(71 / 357)}}{q^{(3 / 14)}}\right) q^{\varepsilon}\right),
\end{aligned}
$$

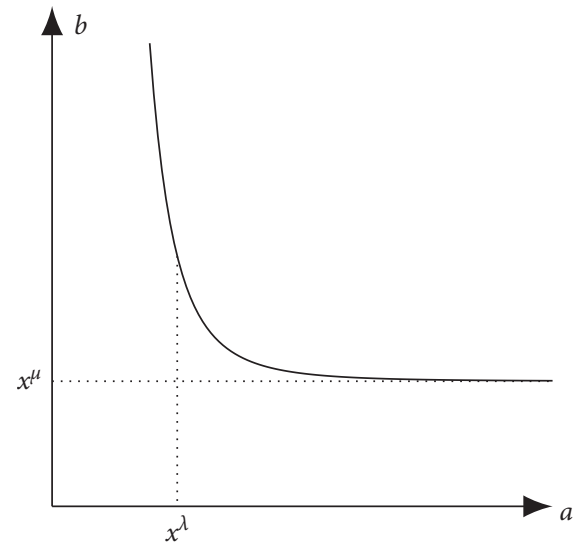

FIgURE 2: Generalized Dirichlet's hyperbola method (proof of Theorem 3 in [7]).

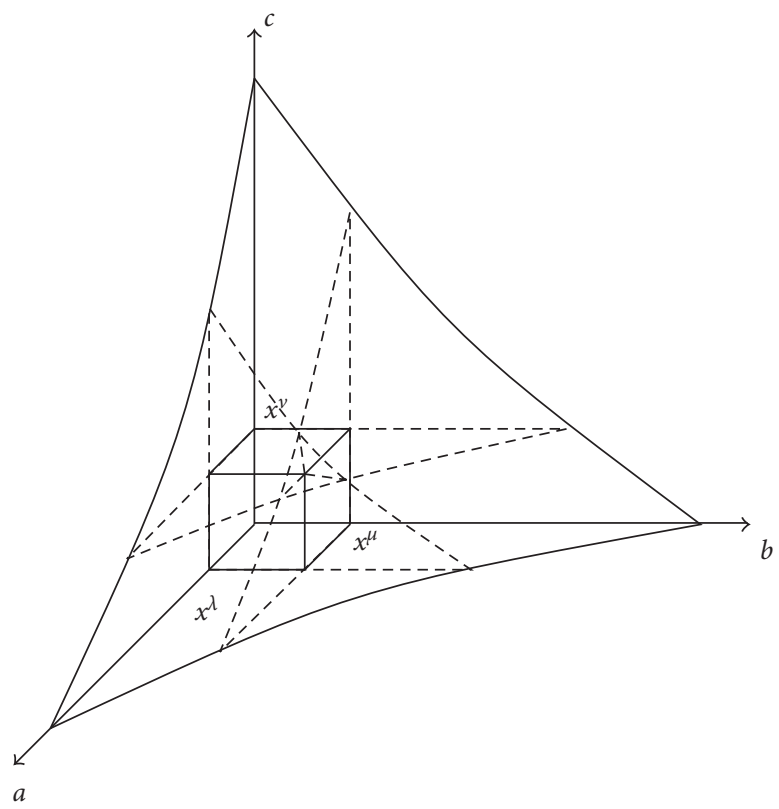

FIgURE 3: Generalized Dirichlet's hyperbola method in three dimensional space (proof of Theorem 2 below).

$$
A_{q}(l)=A_{q}^{\prime}(l)\left(\sum_{\substack{d^{8} e^{9} f^{10} \geq 1 \\(d, e, f, q)=1}} \frac{\mu(d) \mu(f) \mu(e)}{d^{(8 / 3)} e^{3} f^{(10 / 3)}} \sum_{\substack{g \mid e d \\(g, q)=1}} \frac{\mu(g)}{g^{(5 / 3)}}\right),
$$

$$
B_{q}(l)=B_{q}^{\prime}(l)\left(\sum_{\substack{d^{8} e^{9} f^{10} \geq 1 \\(d, e, f, q)=1}} \frac{\mu(d) \mu(f) \mu(e)}{d^{2} e^{(9 / 4)} f^{(5 / 2)}} \sum_{\substack{g \mid e d \\(g, q)=1}} \frac{\mu(g)}{g^{(5 / 4)}}\right),
$$

$$
B_{q}(l)=C_{q}^{\prime}(l)\left(\sum_{\substack{d^{8} e^{9} f^{10} \geq 1 \\(d, e, f, q)=1}} \frac{\mu(d) \mu(f) \mu(e)}{d^{(8 / 5)} e^{(9 / 5)} f^{2}} \sum_{\substack{g \mid e d \\(g, q)=1}} \frac{\mu(g)}{g}\right),
$$

where 
in which $A_{q}^{\prime}(l), B_{q}^{\prime}(l)$, and $C_{q}^{\prime}(l)$ are defined below.

Note that compared with the result in [20], we improve the error term when $x^{(118 / 4029)}<q$.

The key in our proof of Theorem 1 is the following.

Theorem 2. Let $(l, q)=1$, and $\eta, \lambda$ be two parameters such that

$$
\left\{\begin{array}{l}
\frac{1}{10}<\eta<\frac{46}{51} \\
\frac{5}{51}<\lambda<\frac{13}{132}
\end{array}\right.
$$

or

$$
\left\{\begin{array}{l}
\frac{1}{10}<\eta<\frac{67}{660} \\
\frac{13}{132}<\lambda<\frac{1}{10} .
\end{array}\right.
$$

Then, we have

$$
\begin{aligned}
\sum_{\substack{a^{3} b^{4} c^{5} \leq x \\
a^{3} b^{4} c^{5} \equiv l(\bmod q)}} 1= & A_{q}^{\prime}(l) \frac{x^{(1 / 3)}}{q}+B_{q}^{\prime}(l) \frac{x^{(1 / 4)}}{q}+C_{q}^{\prime}(l) \frac{x^{(1 / 5)}}{q} \\
& +O\left(\frac{x^{(1 / 3)-(4 / 3) \eta}}{q^{(1 / 2)-\varepsilon}}+\max \left(x^{\eta}, q^{(1 / 2)}\right) q^{\varepsilon} x^{\lambda}\right),
\end{aligned}
$$

where

$$
\begin{aligned}
A_{q}^{\prime}(l)= & \sum_{b=1}^{\infty} \sum_{c=1}^{\infty} \frac{N_{3}\left(l b^{2} c ; q\right)}{b^{(4 / 3)} c^{(5 / 3)}} \\
B_{q}^{\prime}(l)= & \frac{\phi(q)}{q}\left(5-\frac{5}{4} \int_{1}^{\infty} \frac{t-[t]}{t^{(9 / 4)}} \mathrm{d} t\right)\left(-3-\frac{3}{4} \int_{1}^{\infty} \frac{t-[t]}{t^{(7 / 4)}} \mathrm{d} t\right) \\
& -\sum_{c=1}^{\infty} \frac{N_{4}\left(l c^{3} ; q\right)-((\phi(q)) / q)}{c^{(5 / 4)}} \\
& +\frac{3}{4} \int_{1}^{\infty}\left(\sum_{c=1}^{\infty} \frac{\sum_{a \leq \mu} N_{4}\left(l a c^{3} ; q\right)-((\phi(q)) / q) \mu}{c^{(5 / 4)}}\right) \mu^{-(7 / 4)} \mathrm{d} \mu, \\
C_{q}^{\prime}(l)= & \left.-4-\frac{4}{5} \int_{1}^{\infty} \frac{t-[t]}{t^{(9 / 5)}} \mathrm{d} t\right)\left(-\frac{3}{2}-\frac{3}{5} \int_{1}^{\infty} \frac{t-[t]}{t^{(8 / 5)}} \mathrm{d} t\right) \frac{\phi(q)}{q} \\
& -\frac{4}{5} \int_{1}^{\infty} \frac{\sum_{b \leq \mu} N_{5}(l b ; q)-((\phi(q)) / q) \mu}{\mu^{(8 / 5)}} \mathrm{d} \mu \\
& +N_{5}(l ; q)-\frac{\phi(q)}{q}-\frac{3}{5} \int_{1}^{\infty} \frac{\sum_{a \leq \mu} N_{5}\left(l a^{2} ; q\right)-((\phi(q)) / q)}{\mu^{(8 / 5)}} \mathrm{d} \mu \\
& +\frac{12}{25} \int_{1}^{\infty} \int_{1}^{\infty} \frac{\sum_{b \leq \lambda} \sum_{a \leq \mu} N_{5}\left(l a^{2} b ; q\right)-((\phi(q)) / q) \lambda \mu}{\mu^{(8 / 5)} \lambda^{(9 / 5)}} \mathrm{d} \lambda \mathrm{d} \mu .
\end{aligned}
$$

\section{Some Lemmas}

Before we start the proof, let us give a few lemmas which are needed later.

Lemma 1. For $(l, q)=1$,

$$
\begin{array}{ll}
\sum_{a \leq u} N_{i}(l a ; q)=\frac{\phi(q)}{q} u+O_{\varepsilon}\left(q^{(1 / 2)+\varepsilon}\right), & i=3,4,5, \\
\sum_{a \leq u} N_{i}\left(l a^{2} ; q\right)=\frac{\phi(q)}{q} u+O_{\varepsilon}\left(q^{(1 / 2)+\varepsilon}\right), & i=3,5, \\
\sum_{a \leq u} N_{4}\left(l a^{3} ; q\right)=\frac{\phi(q)}{q} u+O_{\varepsilon}\left(q^{(1 / 2)+\varepsilon}\right) . &
\end{array}
$$

Proof. The first result can be found in Lemma 3 of [21]. Note that

$$
\sum_{a \leq q} N_{i}(l a ; q)=\phi(q), \quad i=3,4,5
$$

The second and third one can be proved in the same way. The proofs of the last three are slightly different. For example, by orthogonal property of additive characters, we have

$$
\sum_{a \leq u} N_{3}\left(l a^{2} ; q\right)=\frac{1}{q} \sum_{a \leq u} \sum_{n=1}^{q} \sum_{x=1}^{q} e\left(\frac{n x^{3}-\ln a^{2}}{q}\right),
$$

$$
\begin{aligned}
& =\frac{1}{q} \sum_{a \leq u} \sum_{n=1}^{q} e\left(\frac{-\ln a^{2}}{q}\right) \sum_{y=1}^{q} e\left(\frac{n y}{q}\right) \sum_{\chi \in G_{3}} \chi(y) \\
& =\frac{1}{q} \sum_{\chi \in G_{3}} \sum_{a \leq u} \sum_{y=1}^{q} \chi(y) \sum_{n=1}^{q} e\left(\frac{n y-\ln a^{2}}{q}\right) \\
& =\sum_{\chi \in G_{3}} \sum_{a \leq u} \sum_{\substack{y \leq q \\
y \equiv l a^{2}(\bmod q)}} \chi(y) \\
& =\sum_{a \leq u} \sum_{\substack{y \leq q \\
y \equiv l a^{2}(\bmod q) \\
(y, q)=1}} 1+\sum_{\substack{\chi \in G_{3} \\
\chi \neq \chi_{0}}} \sum_{\substack{y \leq u \\
y \leq l a^{2}(\bmod q)}} \chi(y) \\
& =\sum_{\substack{a \leq u \\
(a, q)=1}} 1+O\left(\sum_{\substack{\chi \in G_{3} \\
\chi \neq \chi_{0}}}\left|\sum_{a \leq u} \chi^{2}(a)\right|\right) \\
& =\sum_{d \mid q} \mu(d) \sum_{\substack{a \leq u \\
d \mid a}} 1+O\left(\left|G_{3}\right| q^{(1 / 2)+\varepsilon}\right) \\
& =\frac{\phi(q)}{q} u+O\left(q^{(1 / 2)+\varepsilon}\right) \text {, }
\end{aligned}
$$


where $G_{3}$ is the set of all characters $\chi(\bmod q)$ such that $\chi^{3}=\chi_{0}$, the principal character.

Lemma 2. For $q \geq 1$ and $(l, q)=1$,

$$
\sum_{n=1}^{q-1}(n, q)\left|\sum_{b=B+1}^{B+L} \sum_{c=C+1}^{C+M} e\left(\frac{-n l b^{2} c}{q}\right)\right| \ll q \cdot \mathrm{d}(q) \log q .
$$

$$
\begin{aligned}
\sum_{n=1}^{q-1}(n, q)\left|\sum_{b=B+1} \sum_{c=C+1}^{C+L} e\left(\frac{-n l b^{2} c}{q}\right)\right| & =\sum_{d \mid q} d \sum_{n_{l}=1}^{(q / d-1)}\left|\sum_{b=B+1}^{B+L} \sum_{c=C+1}^{C+M} e\left(\frac{-n / l b^{2} c}{(q / d)}\right)\right|, \\
& \ll \sum_{d \mid q} d \sum_{n_{l}=1}^{(q / d-1)} \sum_{b=B+1}^{B+L} \min \left(L, \frac{1}{\left\|\left(n / b^{2}\right) /(q / d)\right\|}\right) \\
& \ll \sum_{d \mid q} d \sum_{b=B+1}^{B+L}\left(\sum_{n_{l} \leq\left(q / L d b^{2}\right)} L+\sum_{\left(q / L d b^{2}\right)<n_{l} \leq(q / d)} \frac{(q / d)}{n / b^{2}}\right) \\
& \ll \sum_{d \mid q} d\left(\frac{q}{d}+\log q \cdot \frac{q}{d}\right), \\
& \ll q \cdot \mathrm{d}(q) \log q .
\end{aligned}
$$

Lemma 3. For $(l, q)=1$,

$$
\begin{gathered}
\sum_{\substack{b \leq x^{\mu} \\
(b, q)=1}} \sum_{\substack{c \leq x^{\nu} \\
(c, q)=1}} \sum_{\substack{a \leq x^{\lambda} \\
a^{3} b^{4} c^{5} \equiv l(\bmod q)}} 1=\frac{\phi(q)}{q^{2}} x^{\lambda+\mu+\nu}+O_{\varepsilon}\left(\frac{x^{\lambda}}{q^{(1 / 2)-\varepsilon}}\right) \\
+O_{\varepsilon}\left(x^{\mu+\nu} q^{\varepsilon}\right) .
\end{gathered}
$$

$$
\begin{aligned}
& \sum_{\substack{b \leq x^{\mu} \\
(b, q)=1}} \sum_{\substack{c \leq x^{\nu} \\
(c, q)=1}} \sum_{\substack{a \leq x^{\lambda} \\
a^{3} b^{4} c^{5} \equiv l(\bmod q)}}=\sum_{b \leq x^{\mu}} \sum_{c \leq x^{\nu}} N_{3}\left(l b^{2} c ; q\right)\left(\frac{x^{\lambda}}{q}+O(1)\right) \\
&=\frac{x^{\lambda}}{q} \sum_{b \leq x^{\mu}} \sum_{c \leq x^{\nu}} N_{3}\left(l b^{2} c ; q\right)+O_{\varepsilon}\left(\sum_{b \leq x^{\mu}} \sum_{c \leq x^{\nu}} N_{3}\left(l b^{2} c ; q\right)\right) .
\end{aligned}
$$

Then, by orthogonal property of additive characters, we obtain

$$
\begin{aligned}
\sum_{b \leq x^{\mu}} \sum_{c \leq x^{\nu}} N_{3}\left(l b^{2} c ; q\right) & =\sum_{b \leq x^{\mu}} \sum_{c \leq x^{\nu}} \frac{1}{q} \sum_{n=1}^{q} \sum_{x=1}^{q} r e\left(\frac{n\left(x^{3}-l b^{2} c\right)}{q}\right) \\
& =\frac{\phi(q)}{q}\left(x^{\mu+\nu}+O(1)\right)+\frac{1}{q} \sum_{n=1}^{q-1} \sum_{b \leq x^{\mu}} \sum_{c \leq x^{\nu}} e\left(\frac{-n l b^{2} c}{q}\right) \sum_{x=1}^{q} r e\left(\frac{n x^{3}}{q}\right) \\
& =\frac{\phi(q)}{q}\left(x^{\mu+\nu}+O(1)\right)+\frac{1}{q} \sum_{n=1}^{q-1} \sum_{b \leq x^{\mu}} \sum_{c \leq x^{\nu}} e\left(\frac{-n l b^{2} c}{q}\right) \sum_{y(\bmod q)} e\left(\frac{n y}{q}\right) \sum_{\chi \in G_{3}} \chi(y) .
\end{aligned}
$$


Interchanging the order of summations and combining Lemma 2 and Eq. (12.48) on page 324 of [24], we have

$$
\begin{aligned}
\sum_{b \leq x^{\mu}} \sum_{c \leq x^{\nu}} N_{3}\left(l b^{2} c ; q\right) & =\frac{\phi(q)}{q}\left(x^{\mu+\nu}+O(1)\right)+O\left(\frac{1}{q} \sum_{\chi \in G_{3}} \sum_{n=1}^{q-1}\left|\sum_{b \leq x^{\mu}} \sum_{c \leq x^{\nu}} e\left(\frac{-n l b^{2} c}{q}\right)\right|(n, q) d(n, q) \sqrt{q}\right) \\
& =\frac{\phi(q)}{q}\left(x^{\mu+\nu}+O(1)\right)+O\left(\frac{d(q)}{\sqrt{q}}\left|G_{3}\right| \sum_{n=1}^{q-1}\left|\sum_{b \leq x^{\mu}} \sum_{c \leq x^{\nu}} e\left(\frac{-n l b^{2} c}{q}\right)\right|(n, q)\right) \\
& =\frac{\phi(q)}{q} x^{\mu+\nu}+O_{\varepsilon}\left(q^{(1 / 2)+\varepsilon}\right) .
\end{aligned}
$$

Finally, we get

$$
\sum_{\substack{b \leq x^{\mu} \\(b, q)=1}} \sum_{\substack{c \leq x^{\nu} \\(c, q)=1}} \sum_{\substack{a \leq x^{\lambda} \\ a^{3} b^{4} c^{5} \equiv l(\bmod q)}} 1=\frac{\phi(q)}{q^{2}} x^{\lambda+\mu+v}+O_{\varepsilon}\left(\frac{x^{\lambda}}{q^{(1 / 2)-\varepsilon}}\right)+O_{\varepsilon}\left(x^{\mu+v} q^{\varepsilon}\right) .
$$

By definition of $N_{3}(n ; q), N_{3}(n ; q) \ll_{\varepsilon} q^{\varepsilon}$ (see Lemma 2.2 in $[25])$.

Lemma 4. If we define

$$
S(a, b ; q)=\sum_{n=1}^{q} 1 e\left(\frac{a n^{3}+b \bar{n}^{4}}{q}\right)
$$

then we have $|S(a, b ; q)| \ll(a, b, q)^{(1 / 2)} q^{(1 / 2)+\varepsilon}$.

Proof. Following much the same way as Lemma 4 of [21], we first suppose $q=r s$ with $(r, s)=1$. By the "reciprocity" formula $s \bar{s}+r \bar{r} \equiv 1(\bmod q)$, where $s \bar{s} \equiv 1(\bmod r)$ and $r \bar{r} \equiv 1(\bmod s)$, and the additive multiplicity of exponential function

$$
e\left(\frac{a n^{3}+b \bar{n}^{4}}{q}\right)=e\left(\frac{a \bar{s} n^{3}+b \overline{s n}^{4}}{r}\right) e\left(\frac{a \bar{r} n^{3}+b \overline{r n}^{4}}{s}\right),
$$

with $n=s x+r y, \quad 1 \leq x \leq r, \quad 1 \leq y \leq s, \quad(x, r)=1$, and $(y, s)=1$, it follows that

$$
\begin{aligned}
S(a, b ; q) & =\sum_{x=1}^{r}, \sum_{y=1}^{s} l e\left(\frac{a \bar{s} x^{3}+b \bar{s} x^{4}}{r}\right) e\left(\frac{a \bar{r} y^{3}+b \bar{r} \bar{y}^{4}}{s}\right) \\
& =S(a \bar{s}, b \bar{s} ; r) S(a \bar{r}, b \bar{r} ; s) .
\end{aligned}
$$

Now we just need to discuss the argument in the following cases:

(I) Prime moduli $q=p$ case. Now Theorem 2 obtained by Moreno and Moreno [26], which is a special form of the Bombieri-Weil bound [27], implies

$$
|S(a, b ; p)| \leq 10(a, b, p)^{(1 / 2)} p^{(1 / 2)},
$$

provided that $\left(\left(a x^{7}+b\right) / x^{4}\right)$ is not the form of $h^{p}(x)-h(x)$ with $h(x) \in \bar{F}_{p}[x]$, where $\bar{F}_{p}$ is the algebraic closure of $F_{p}$. Let

$$
\frac{a x^{7}+b}{x^{4}}=\frac{f^{p}(x)}{g^{p}(x)}-\frac{f(x)}{g(x)}
$$

with $f(x), g(x) \in \bar{F}_{p}[x]$ and $(f(x), g(x))=1$. Then, we have $g^{p}(x) \mid x^{4}$, derived from

$g^{p}(x)\left(a x^{7}+b\right)=x^{4}\left(f^{p}(x)-g^{p-1}(x) f(x)\right)$.

This is impossible if $p>4$, by comparing the degrees of both sides of the above. If $p \leq 4$, the validity of (26) can be easily checked.

(II) Prime power moduli $q=p^{\beta}$ case with $\beta>1$. Obviously, we only need to consider it with the assumption $(a, b, p)=1$. Following the proofs of Lemma 12.2 and 12.3 in [24] with the equation of $S(a, b ; q)$, we obtain

$$
\begin{aligned}
S\left(a, b ; p^{2 \alpha}\right) & =p^{\alpha} \sum_{\substack{y=1 \\
g_{\prime}(y) \equiv 0\left(\bmod p^{\alpha}\right)}}^{p^{\alpha}} 1 e\left(\frac{g(y)}{p^{2 \alpha}}\right) \\
S\left(a, b ; p^{2 \alpha+1}\right) & =p^{\alpha} \sum_{\substack{y=1 \\
g_{l}(y) \equiv 0\left(\bmod p^{\alpha}\right)}}^{p^{\alpha}} 1 e\left(\frac{g(y)}{p^{2 \alpha+1}}\right) G_{p}(y),
\end{aligned}
$$

where

$$
\begin{aligned}
g(y) & =\frac{a y^{7}+b}{y^{4}}, \\
G_{p}(y) & =\sum_{z=1}^{p} e\left(\frac{h(y) z^{2}+g^{\prime}(y) p^{-\alpha} z}{p}\right),
\end{aligned}
$$

with $h(y)=\left(g^{\prime \prime}(y) / 2\right)$. 
Note that $g^{\prime}(y)=\left(\left(3 a y^{10}-4 b y^{3}\right) / y^{8}\right) \quad$ and $h(y)=\left(\left(3 a y^{11}+10 b y^{4}\right) / y^{10}\right)$. Now we concentrate on the number of solutions of congruence equation $g^{\prime}(y) \equiv 0\left(\bmod p^{\alpha}\right)$ with $(y, p)=1$.

$\beta=2 \alpha$ with $\alpha \geq 1$. Then, the congruence equation is

$$
3 a y^{7}-4 b \equiv 0\left(\bmod p^{\alpha}\right) \text {. }
$$

If $(b, p)=p$, then $(a, p)=1$. Relying on the properties of indices, we deduce that (32) has no solution when $p>3$ and one solution when $p=3$. Next, we assume $(b, p)=1$. If $p=2$ and $4 \| a$, then (32) has at most seven solutions. And if $p>3$ and $(a, p)=1$, (32) also has at most seven solutions. Then, we have

$$
\left|S\left(a, b ; p^{2 \alpha}\right)\right| \leq 7 p^{\alpha}, \quad \text { if }(a, b, p)=1,
$$

where $\beta=2 \alpha+1$ with $\alpha \geqslant 1$. Firstly, in the same way, if $(b, p)=p$, by the analysis in the case $\beta=2 \alpha$, the sum in (30) is empty unless $p=3$ in which case one has $\left|S\left(a, b ; 3^{2 \alpha+1}\right)\right| \leq 3^{\alpha+1}$. Now suppose $(b, p)=1$; according to Chapter 3 in [24], we know if $p \nmid 2 h(y)$, then we have $\left|G_{p}(y)\right| \leq p^{(1 / 2)}$. Therefore, if $p \neq 2,7$ (for otherwise $p \mid 3 a y^{7}+$ $10 b$ and $p \mid 3 a y^{7}-4 b$ imply $p \mid 14 b$, a contradiction), we have $\left|G_{p}(y)\right| \leq p^{(1 / 2)}$; hence, $\left|S\left(a, b ; p^{2 \alpha+1}\right)\right| \leq 7 p^{\alpha+(1 / 2)}$ as there are at most seven solutions to (32). If $p=2$ or $7,\left|G_{p}(y)\right| \leq 7$, and hence $\left|S\left(a, b ; p^{2 \alpha+1}\right)\right| \leq 49 p^{\alpha}$.
In any case, we have

$$
\left|S\left(a, b ; p^{2 \alpha+1}\right)\right| \leq 49 p^{(\alpha+1) / 2}, \quad \text { if }(a, b, p)=1 .
$$

Combining (25), (26), (33), and (34), we finally obtain

$$
|S(a, b ; q)| \leq 49^{\omega(q)}(a, b, q)^{(1 / 2)} q^{(1 / 2)} \ll(a, b, q)^{(1 / 2)} q^{(1 / 2)+\varepsilon},
$$

which completes the proof of Lemma 4.

By applying Lemma 4, we have the following.

Lemma 5. For $(l, q)=1$,

$$
\begin{aligned}
N_{A, B}(K, L):= & \#\left\{(a, b): a^{3} b^{4} \equiv l(\bmod q), A<a \leq A\right. \\
& +K, B<b \leq B+L\} \\
= & \frac{\phi(q) K L}{q^{2}}+O\left(\frac{K+L}{q^{(1 / 2)-\varepsilon}}+q^{(1 / 2)+\varepsilon}\right) .
\end{aligned}
$$

Proof. Note that if $a^{3} b^{4} \equiv l(\bmod q)$, then $a^{4} b^{4} \equiv l a(\bmod q)$ and $b \equiv l(\overline{a b})^{3}(\bmod q)$. So,

$$
N_{A, B}(K, L)=\#\left\{(a, b, w): a \equiv \bar{l} w^{4}(\bmod q), b \equiv l \bar{w}^{3}(\bmod q), A<a \leq A+K, B<b \leq B+L, 1 \leq w \leq q,(w, q)=1\right\}
$$

The remaining part of the proof is similar to Theorem 4 in [21].

\section{Proof of Theorem 2}

Consider three positive parameters $\lambda, \mu$, and $\nu$. By extending the construction from Montgomery and Vaughan [23] as shown in Figure 3, we have

$$
\begin{aligned}
& \sum_{\substack{a^{3} b^{4} c^{5} \leq x \\
a^{3} b^{4} c^{5} \equiv l(\bmod q)}} 1=\sum_{\substack{a \leq x^{\lambda} \\
(a, q)=1}} 1+\sum_{\substack{b \leq x^{4} \\
b^{4} c^{5} \leq\left(x / a^{3}\right) \\
b^{4} c^{5} \equiv l a^{3}(\bmod q)}} \sum_{\substack{a^{3} c^{5} \leq\left(x / b^{4}\right) \\
(b, q)=1 \\
a^{3} c^{5} \equiv \bar{l} b^{4}(\bmod q)}} 1+\sum_{\substack{c \leq x^{v} \\
(c, q)=1}} 1-\sum_{\substack{a^{3} b^{4} \leq\left(x / c^{5}\right) \\
a^{3} b^{4} \equiv l \bar{c} c^{5}(\bmod q)}} \sum_{\substack{a \leq x^{\lambda} \\
(a, q)=1}} \sum_{\substack{b \leq x^{\mu} \\
(b, q)=1}} 1 \\
& -\sum_{\substack{b \leq x^{\mu} \\
(b, q)=1}} \sum_{\substack{c \leq x^{\nu} \\
(c, q)=1}} \sum_{\substack{a^{3} \leq\left(x /\left(b^{4} c^{5}\right)\right) \\
a^{3} \equiv l \overline{b^{4} c^{5}}(\bmod q)}} 1-\sum_{\substack{a \leq x^{\lambda} \\
(a, q)=1}} \sum_{\substack{c \leq x^{\nu} \\
(c, q)=1}} \sum_{\substack{b^{4} \leq\left(x /\left(a^{3} c^{5}\right)\right) \\
b^{4} \equiv l \bar{a}^{3} c^{5}(\bmod q)}} 1+\sum_{\substack{a \leq x^{\lambda} \\
(a, q)=1}} \sum_{\substack{b \leq x^{\mu} \\
(b, q)=1}} 1 \\
& +\sum_{\substack{a>x^{\lambda} \\
(a, q)=1}} \sum_{\substack{b>x^{\mu} \\
(b, q)=1}} \sum_{\substack{c>x^{v} \\
a^{3} b^{4} c^{5} \equiv l(\bmod q) \\
a^{3} b^{4} c^{5} \equiv l(\bmod q)}} 1 \\
& :=T_{1}+T_{2}+T_{3}-T_{4}-T_{5}-T_{6}+T_{7}+T_{8} \text {. }
\end{aligned}
$$


First, we estimate $T_{3}$.

$$
\begin{aligned}
& T_{3}=\sum_{\substack{c \leq x^{v} \\
(c, q)=1}} \sum_{\substack{a^{3} b^{4} \leq\left(x / c^{5}\right) \\
a^{3} b^{4} \equiv l c^{5}(\bmod q)}} 1, \\
& =\sum_{\substack{c \leq x^{v} \\
(c, q)=1}} \sum_{\substack{b \leq x^{\mu_{1}} \\
(b, q)=1}} \sum_{\substack{a^{3} \leq\left(x /\left(b^{4} c^{5}\right)\right) \\
a^{3} \equiv l b^{4} c^{5}(\bmod q)}} 1+\sum_{\substack{c \leq x^{v} \\
(c, q)=1}} \sum_{\substack{a \leq x^{\lambda_{1}} \\
(a, q)=1}} \sum_{\substack{b^{4} \leq\left(x /\left(a^{3} c^{5}\right)\right) \\
b^{4} \equiv l \bar{a}^{3} c^{5}(\bmod q)}} 1 \\
& -\sum_{\substack{c \leq x^{v} \\
(c, q)=1}} \sum_{\substack{a \leq x^{\lambda_{1}} \\
(a, q)=1}} \sum_{\substack{b \leq x^{\mu_{1}} \\
b^{4} \equiv l a^{3} c^{5}(\bmod q)}} 1+\sum_{\substack{c \leq x^{v} \\
(c, q)=1}} \sum_{\substack{a>\lambda^{\lambda_{1}} \\
a^{3} b^{4} \leq\left(x / c^{5}\right)}} \sum_{\substack{b>x^{\mu_{1}} \\
a^{3} b^{4} \equiv l c^{5}(\bmod q)}} 1 \\
& :=T_{31}+T_{32}-T_{33}+T_{34} \text {. }
\end{aligned}
$$

For $T_{33}$, we have

$$
T_{33}=\frac{\phi(q)}{q^{2}} x^{\lambda_{1}+\mu_{1}+v}+O\left(\frac{x^{\lambda_{1}}}{q^{(1 / 2)-\varepsilon}}\right)+O\left(x^{\mu_{1}+v} q^{\varepsilon}\right),
$$

by Lemma 3 .

Then, we estimate $T_{31}$ and $T_{32}$. For $T_{31}$, we know

$$
\begin{aligned}
T_{31}= & \sum_{c \leq x^{\nu}} \sum_{b \leq x^{\mu_{1}}} N_{3}\left(l b^{2} c ; q\right)\left(\frac{x^{(1 / 3)}}{b^{(4 / 3)} c^{(5 / 3)} q}+O(1)\right) \\
= & \sum_{c \leq x^{\nu}} \frac{1}{c^{(5 / 3)}}\left(\sum_{b=1}^{\infty} \frac{N_{3}\left(l b^{2} c ; q\right)}{b^{(4 / 3)}}\right) \frac{x^{(1 / 3)}}{q}-\sum_{c \leq x^{\nu}} \frac{1}{c^{(5 / 3)}} \frac{3 \phi(q)}{q^{2}} x^{(1 / 3)-(1 / 3) \mu_{1}} \\
& +O\left(\frac{x^{(1 / 3)-(1 / 3) \mu_{1}}}{q^{(1 / 2)-\varepsilon}} \sum_{c \leq x^{\nu}} \frac{1}{c^{(5 / 3)}}\right)+O\left(x^{\mu_{1}+v} q^{\varepsilon}\right) .
\end{aligned}
$$

The first term in the above formula is

$$
\begin{gathered}
\frac{x^{(1 / 3)}}{q} \sum_{b=1}^{\infty} \frac{1}{b^{(4 / 3)}}\left(\sum_{c=1}^{\infty} \frac{N_{3}\left(l b^{2} c ; q\right)}{c^{(5 / 3)}}\right)-\frac{3}{2} \frac{\phi(q)}{q^{2}} x^{(1 / 3)-(2 / 3) \nu} \sum_{b=1}^{\infty} \frac{1}{b^{(4 / 3)}}+O\left(\frac{x^{(1 / 3)-(5 / 3) \nu}}{q^{1 / 2-\varepsilon}}\right) \\
=\frac{x^{(1 / 3)}}{q} \sum_{b=1}^{\infty} \sum_{c=1}^{\infty} \frac{N_{3}\left(l b^{2} c ; q\right)}{b^{(4 / 3)} c^{(5 / 3)}}-\frac{3}{2} \frac{\phi(q)}{q^{2}} x^{(1 / 3)-(2 / 3) \nu} \sum_{b=1}^{\infty} \frac{1}{b^{(4 / 3)}}+O\left(\frac{x^{(1 / 3)-(5 / 3) \nu}}{q^{(1 / 2)-\varepsilon}}\right) .
\end{gathered}
$$

In order to simplify our final result, by using Euler's summation formula which can be found in
Theorem 3.2 in [28], the constant $\sum_{b=1}^{\infty}\left(1 / b^{(4 / 3)}\right)$ can be rewritten as 


$$
\begin{aligned}
\sum_{b=1}^{\infty} \frac{1}{b^{(4 / 3)}} & =\lim _{x \longrightarrow \infty} \sum_{b \leqslant x} \frac{1}{b^{(4 / 3)}} \\
& =\lim _{x \rightarrow \infty}\left(-3 x^{-(1 / 3)}+4-\frac{4}{3} \int_{1}^{\infty} \frac{t-[t]}{t^{(7 / 3)}} \mathrm{d} t+O\left(x^{-(4 / 3)}\right)\right) \\
& =4-\frac{4}{3} \int_{1}^{\infty} \frac{t-[t]}{t^{(7 / 3)}} \mathrm{d} t \\
& =6-2 \int_{1}^{\infty} \frac{t-[t]}{t^{(7 / 3)}} \mathrm{d} t .
\end{aligned}
$$

$$
\begin{aligned}
T_{31}= & \frac{x^{(1 / 3)}}{q} \sum_{b=1}^{\infty} \sum_{c=1}^{\infty} \frac{N_{3}\left(l b^{2} c ; q\right)}{b^{(4 / 3)} c^{(5 / 3)}}-\left(6-2 \int_{1}^{\infty} \frac{t-[t]}{t^{(7 / 3)}} \mathrm{d} t\right) \frac{\phi(q)}{q} x^{(1 / 3)-(2 / 3) v} \\
& -\sum_{c \leq x^{\nu}} \frac{1}{c^{(5 / 3)}} \frac{3 \phi(q)}{q^{2}} x^{(1 / 3)-(1 / 3) \mu_{1}}+O\left(\frac{x^{(1 / 3)-(5 / 3) v}}{q^{(1 / 2)-\varepsilon}}\right)+O\left(\frac{x^{(1 / 3)-(4 / 3) \mu_{1}}}{q^{(1 / 2)-\varepsilon}}+x^{\mu_{1}+v} q^{\varepsilon}\right)
\end{aligned}
$$

Next, we deal with $T_{32}$.

$$
\begin{aligned}
& T_{32}=\sum_{\substack{c \leq x^{v} \\
(c, q)=1}} \sum_{\substack{a \leq x^{\lambda_{1}} \\
(a, q)=1}} \sum_{\substack{b^{4} \leq\left(x /\left(a^{3} c^{5}\right)\right) \\
b^{4} \equiv \overline{a^{3} c^{5}}(\bmod q)}} 1 \\
& =\frac{x^{(1 / 4)}}{q} \sum_{c \leq x^{\nu}} \frac{1}{c^{(5 / 4)}} \sum_{a \leq x^{\lambda_{1}}} \frac{N_{4}\left(l a c^{3} ; q\right)}{a^{(3 / 4)}}+O\left(x^{\nu+\lambda_{1}} q^{\varepsilon}\right) \\
& =\frac{x^{(1 / 4)}}{q} \sum_{c \leq x^{\nu}} \frac{1}{c^{(5 / 4)}}\left(\sum_{a \leq x^{\lambda_{1}}} \frac{N_{4}\left(l a c^{3} ; q\right)-((\phi(q)) / q)}{a^{(3 / 4)}}+\frac{\phi(q)}{q} \sum_{a \leq x^{\lambda_{1}}} \frac{1}{a^{(3 / 4)}}\right)+O\left(x^{\nu+\lambda_{1}} q^{\varepsilon}\right) \\
& =\frac{x^{(1 / 4)}}{q} \sum_{c \leq x^{\nu}} \frac{1}{c^{(5 / 4)}}\left(\int_{1}^{x^{\lambda_{1}}} \frac{1}{\mu^{(3 / 4)}} d\left(\sum_{a \leq \mu} N_{4}\left(l a c^{3} ; q\right)-\frac{\phi(q)}{q} \mu\right)+\frac{\phi(q)}{q}\left(4 x^{(1 / 4) \lambda_{1}}-3-\frac{3}{4} \int_{1}^{\infty} \frac{t-[t]}{t^{(7 / 4)}} \mathrm{d} t+O\left(x^{-\frac{3}{4} \lambda_{1}}\right)\right)\right) \\
& +O\left(x^{\nu+\lambda_{1}} q^{\varepsilon}\right) \\
& =\frac{x^{(1 / 4)}}{q} \sum_{c \leq x^{\nu}} \frac{1}{c^{(5 / 4)}}\left(O\left(\frac{q^{(1 / 2)-\varepsilon}}{x^{(3 / 4) \lambda_{1}}}\right)-N_{4}\left(l c^{3} ; q\right)+\frac{\phi(q)}{q}+\frac{3}{4} \int_{1}^{\infty} \frac{\sum_{a \leq \mu} N_{4}\left(l a c^{3} ; q\right)-((\phi(q)) / q) \mu}{\mu^{(7 / 4)}} \mathrm{d} \mu\right) \\
& +\frac{\phi(q)}{q^{2}} x^{(1 / 4)} \sum_{c \leq x^{\nu}} \frac{1}{c^{(5 / 4)}}\left(4 x^{(1 / 4)} \lambda_{1}-3-\frac{3}{4} \int_{1}^{\infty} \frac{t-[t]}{t^{(7 / 4)}} \mathrm{d} t+O\left(x^{-(3 / 4) \lambda_{1}}\right)\right)+O\left(x^{\nu+\lambda_{1}} q^{\varepsilon}\right) .
\end{aligned}
$$


If we let $F_{c}(\mu)=\sum_{a \leq \mu} N_{4}\left(l a c^{3} ; q\right)-((\phi(q)) / q) \mu$, then the first term in the above formula is

$$
\begin{aligned}
& -\frac{x^{(1 / 4)}}{q} \sum_{c \leq x^{\nu}} \frac{N_{4}\left(l c^{3} ; q\right)-((\phi(q)) / q)}{c^{5 / 4}}+\frac{3}{4} \frac{x^{(1 / 4)}}{q} \int_{1}^{\infty} \frac{\sum_{c \leq x^{\nu}}\left(F_{c}(\mu) / c^{(5 / 4)}\right)}{\mu^{(7 / 4)}} d \mu+O\left(\frac{x^{(1 / 4)-(3 / 4) \lambda_{1}}}{q^{(1 / 2)-\varepsilon}}\right) \\
& =-\frac{x^{(1 / 4)}}{q} \sum_{c=1}^{\infty} \frac{N_{4}\left(l c^{3} ; q\right)-((\phi(q)) / q)}{c^{(5 / 4)}}+O\left(\frac{x^{(1 / 4)-(5 / 4) \nu}}{q^{(1 / 2)-\varepsilon}}\right)+\frac{3}{4} \frac{x^{(1 / 4)}}{q} \int_{1}^{\infty}\left(\sum_{c=1}^{\infty} \frac{F_{c}(\mu)}{c^{(5 / 4)}}\right) \mu^{-(7 / 4)} \mathrm{d} \mu \\
& \quad+O\left(\frac{x^{(1 / 4)-(3 / 4) \lambda_{1}}}{q^{(1 / 2)-\varepsilon}}\right)+O\left(\frac{x^{(1 / 4)-(5 / 4) \nu}}{q^{(1 / 2)-\varepsilon}}\right) \\
& =\frac{x^{(1 / 4)}}{q}\left(-\sum_{c=1}^{\infty} \frac{N_{4}\left(l c^{3} ; q\right)-((\phi(q)) / q)}{c^{(5 / 4)}}+\frac{3}{4} \frac{x^{(1 / 4)}}{q} \int_{1}^{\infty}\left(\sum_{c=1}^{\infty} \frac{F_{c}(\mu)}{c^{(5 / 4)}}\right) \mu^{-(7 / 4)} \mathrm{d} \mu\right)+O\left(\frac{x^{(1 / 4)-(3 / 4) \lambda_{1}}}{q^{(1 / 2)-\varepsilon}}\right)+O\left(\frac{x^{(1 / 4)-(5 / 4) \nu}}{q^{(1 / 2)-\varepsilon}}\right) .
\end{aligned}
$$

And the second term is

$$
\begin{aligned}
& \frac{\phi(q)}{q^{2}} x^{(1 / 4)}\left(-4 x^{-(1 / 4) v}+5-\frac{5}{4} \int_{1}^{\infty} \frac{t-[t]}{t^{(9 / 4)}} \mathrm{d} t+O\left(x^{-(1 / 4) v}\right)\right)\left(4 x^{(1 / 4) \lambda_{1}}-3-\frac{3}{4} \int_{1}^{\infty} \frac{t-[t]}{t^{(7 / 4)}} \mathrm{d} t+O\left(x^{-(3 / 4) \lambda}\right)\right) \\
& =4 \frac{\phi(q)}{q^{2}} x^{(1 / 4)+(1 / 4) \lambda_{1}} \sum_{c \leq x^{\nu}} \frac{1}{c^{(5 / 4)}}+\frac{\phi(q)}{q^{2}} x^{(1 / 4)-(1 / 4) v}\left(12+3 \int_{1}^{\infty} \frac{t-[t]}{t^{(7 / 4)}} \mathrm{d} t\right) \\
& +\frac{\phi(q)}{q^{2}} x^{(1 / 4)}\left(5-\frac{5}{4} \int_{1}^{\infty} \frac{t-[t]}{t^{(9 / 4)}} \mathrm{d} t\right)\left(-3-\frac{3}{4} \int_{1}^{\infty} \frac{t-[t]}{t^{(7 / 4)}} \mathrm{d} t\right)+O\left(\frac{\phi(q)}{q^{2}} x^{(1 / 4)-(5 / 4) \nu}\right)+O\left(\frac{\phi(q)}{q^{2}} x^{(1 / 4)-(3 / 4) \lambda_{1}}\right) .
\end{aligned}
$$

So, we can get

$$
\begin{aligned}
T_{32}= & \frac{x^{(1 / 4)}}{q}\left[\frac{\phi(q)}{q}\left(5-\frac{5}{4} \int_{1}^{\infty} \frac{t-[t]}{t^{(9 / 4)}} \mathrm{d} t\right)\left(-3-\frac{3}{4} \int_{1}^{\infty} \frac{t-[t]}{t^{(7 / 4)}} \mathrm{d} t\right)-\sum_{c=1}^{\infty} \frac{N_{4}\left(l c^{3} ; q\right)-((\phi(q)) / q)}{c^{(5 / 4)}}\right. \\
& \left.+\frac{3}{4} \frac{x^{(1 / 4)}}{q} \int_{1}^{\infty}\left(\sum_{c=1}^{\infty} \frac{F_{c}(\mu)}{c^{(5 / 4)}}\right) \mu^{-(7 / 4)} d \mu\right]+4 \frac{\phi(q)}{q^{2}} x^{(1 / 4)+(1 / 4) \lambda_{1}} \sum_{c \leq x^{\nu}} \frac{1}{c^{(5 / 4)}}+\frac{\phi(q)}{q^{2}} x^{(1 / 4)-(1 / 4) v}\left(12+3 \int_{1}^{\infty} \frac{t-[t]}{t^{(7 / 4)}} \mathrm{d} t\right) \\
& +O\left(\frac{\phi(q)}{q^{2}} x^{(1 / 4)-(5 / 4) \nu}\right)+O\left(\frac{\phi(q)}{q^{2}} x^{(1 / 4)-(3 / 4) \lambda_{1}}\right)+O\left(\frac{x^{(1 / 4)-(3 / 4) \lambda_{1}}}{q^{(1 / 2)-\varepsilon}}\right)+O\left(\frac{x^{(1 / 4)-(5 / 4) \nu}}{q^{(1 / 2)-\varepsilon}}\right)+O\left(x^{\nu+\lambda_{1}} q^{\varepsilon}\right) .
\end{aligned}
$$

And for $T_{34}$, following closely Chan [21] as shown in Figure 2 instead of Dirichlet's hyperbola method shown in Figure 1, we just need to divide the interval $\{(a, b)$ : $\left.a>x^{n}, b>x^{m}, a^{3} b^{4} \leq x\right\}$ in the same way. Note that the sum

$$
\sum_{\substack{a>x^{\lambda_{1}} \\ a^{3} b^{4} \leq\left(x / c^{5}\right)}} \sum_{\substack{b>x^{\mu_{1}} \\ a^{3} b^{4} \equiv l c^{5}(\bmod q)}} 1
$$


can be estimated with the help of asymptotic formula given at the end of page 101 in [21]; then, using Lemma 5, we can get

$$
\begin{aligned}
T_{34}= & \sum_{c \leq x^{\nu}} \frac{1}{c^{(5 / 3)}} \cdot \frac{3 \phi(q)}{q^{2}} x^{(1 / 3)-(1 / 3) \mu_{1}}-\sum_{c \leq x^{\nu}} \frac{1}{c^{(5 / 4)}} \cdot \frac{3 \phi(q)}{q^{2}} x^{(1 / 4)-(1 / 4) \lambda_{1}}+\frac{\phi(q)}{q^{2}} x^{\lambda_{1}+\mu_{1}+\nu} \\
& +O\left(\frac{x^{(1 / 3)-(1 / 3) \mu_{1}}}{2^{J_{1}} \cdot q} \sum_{c \leq x^{\nu}} \frac{1}{c^{(5 / 4)}}+2^{J_{1}} q^{(1 / 2)+\varepsilon} x^{\nu} \log x\right)
\end{aligned}
$$

where $2^{J_{1}}$ satisfies $\left(X^{\mu_{1}} /\left(2^{J_{1}} q^{(1 / 2)}\right)\right) \ll 1$.
Picking $\lambda_{1}$ to have the same size as $\mu_{1}$ and combining the previous results, we have

$$
\begin{aligned}
T_{3}= & \frac{x^{(1 / 3)}}{q} \sum_{b=1}^{\infty} \sum_{c=1}^{\infty} \frac{N_{3}\left(l b^{2} c ; q\right)}{b^{(4 / 3)} c^{(5 / 3)}}-\left(6-2 \int_{1}^{\infty} \frac{t-[t]}{t^{(7 / 3)}} \mathrm{d} t\right) \frac{\phi(q)}{q} x^{(1 / 3)-(2 / 3) v}+\frac{\phi(q)}{q^{2}} x^{(1 / 4)-(1 / 4) v}\left(12+3 \int_{1}^{\infty} \frac{t-[t]}{t^{(7 / 4)}} \mathrm{d} t\right) \\
& +\frac{x^{(1 / 4)}}{q}\left[\frac{\phi(q)}{q}\left(5-\frac{5}{4} \int_{1}^{\infty} \frac{t-[t]}{t^{(9 / 4)}} \mathrm{d} t\right)\left(-3-\frac{3}{4} \int_{1}^{\infty} \frac{t-[t]}{t^{(7 / 4)}} \mathrm{d} t\right)-\sum_{c=1}^{\infty} \frac{N_{4}\left(l c^{3} ; q\right)-((\phi(q)) / q)}{c^{(5 / 4)}}\right. \\
& \left.+\frac{3}{4} \frac{x^{(1 / 4)}}{q} \int_{1}^{\infty}\left(\sum_{c=1}^{\infty} \frac{F_{c}(\mu)}{c^{(5 / 4)}}\right) \mu^{-(7 / 4)} \mathrm{d} \mu\right] \\
& +O\left(x^{\mu_{1}+v} q^{\varepsilon}+2^{J_{1}} q^{(1 / 2)+\varepsilon} x^{\nu}+\frac{x^{(1 / 3)-(5 / 3) v}}{q^{(1 / 2)-\varepsilon}}+\frac{x^{(1 / 4)-(5 / 4) v}}{q^{(1 / 2)-\varepsilon}}+\frac{x^{(1 / 4)-(3 / 4) \lambda_{1}}}{q^{(1 / 2)-\varepsilon}}+\frac{x^{(1 / 3)-(4 / 3) \mu_{1}}}{q^{(1 / 2)-\varepsilon}}\right) .
\end{aligned}
$$

For $T_{2}$, in the same way as $T_{3}$, we have

$$
\begin{aligned}
& T_{2}=\sum_{\substack{b \leq x^{\mu} \\
(b, q)=1}} \sum_{\substack{a^{3} c^{5} \leq\left(x / b^{4}\right) \\
a^{3} c^{5} \equiv l \overline{b^{4}}(\bmod q)}} 1 \\
& =\sum_{\substack{b \leq x^{\mu} \\
(b, q)=1}} \sum_{\substack{c \leq x^{\mu_{2}} \\
(c, q)=1}} \sum_{\substack{a^{3} \leq\left(x / b^{4} c^{5}\right) \\
a^{3} \equiv \overline{b^{4} c^{5}}(\bmod q)}} 1+\sum_{\substack{b \leq x^{\mu} \\
(b, q)=1}} \sum_{\substack{a \leq x^{\lambda_{2}} \\
(a, q)=1}} \sum_{\substack{c^{5} \leq\left(x / a^{3} b^{4}\right) \\
c^{5} \equiv l \overline{a^{3} b^{4}}(\bmod q)}} 1 \\
& -\sum_{\substack{b \leq x^{\mu} \\
(b, q)=1}} \sum_{\substack{a \leq x^{\lambda_{2}} \\
(a, q)=1}} \sum_{\substack{c \leq x^{\mu_{2}} \\
b^{4} \equiv l a^{3} c^{5}(\bmod q)}} 1+\sum_{\substack{b \leq x^{\mu} \\
(b, q)=1}} \sum_{\substack{a>x^{\lambda_{2}} \\
a^{3} c^{5} \leq\left(x / b^{4}\right)}} \sum_{\substack{c>x^{\mu_{2}} \\
a^{3} c^{5} \equiv l b^{4}(\bmod q)}} 1 \\
& :=T_{21}+T_{22}-T_{23}+T_{24} \text {. }
\end{aligned}
$$


And we can obtain

$$
\begin{aligned}
T_{21}= & \sum_{b \leq x^{\mu}} \frac{1}{b^{(4 / 3)}}\left(\sum_{c=1}^{\infty} \frac{N_{3}\left(l b^{2} c ; q\right)}{c^{(5 / 3)}}\right) \frac{x^{(1 / 3)}}{q}-\frac{3}{2} \frac{\phi(q)}{q^{2}} \sum_{b \leq x^{\mu}} \frac{1}{b^{(4 / 3)}} x^{(1 / 3)-(2 / 3) \mu_{2}}+O\left(x^{\mu+\mu_{2}} q^{\varepsilon}+\frac{x^{(1 / 3)-(5 / 3) \mu_{2}}}{q^{(1 / 2)-\varepsilon}} \sum_{b \leq x^{\mu}} \frac{1}{b^{(4 / 3)}}\right) \\
T_{23}= & \frac{\phi(q)}{q^{2}} x^{\lambda_{2}+\mu_{2}+\mu}+O\left(\frac{x^{\lambda_{2}}}{q^{(1 / 2)-\varepsilon}}\right)+O\left(x^{\mu_{2}+\mu} q^{\varepsilon}\right), \\
T_{24}= & \sum_{b \leq x^{\mu}} \frac{1}{b^{(4 / 3)}} \cdot \frac{3}{2} \frac{\phi(q)}{q^{2}} x^{(1 / 3)-(2 / 3) \mu_{2}}-\sum_{b \leq x^{\mu}} \frac{1}{b^{(4 / 5)}} \cdot \frac{5}{2} \frac{\phi(q)}{q^{2}} x^{(1 / 5)-(2 / 5) \lambda_{2}}+\frac{\phi(q)}{q^{2}} x^{\lambda_{2}+\mu_{2}+\mu} \\
& +O\left(\frac{x^{(1 / 3)-(2 / 3) \mu_{2}}}{2^{J_{2}} \cdot q} \sum_{b \leq x^{\mu}} \frac{1}{b^{(4 / 5)}}+2^{J_{2}} q^{(1 / 2)+\varepsilon} x^{\mu} \log x\right) .
\end{aligned}
$$

For $T_{22}$, again in the same way as the proof of $T_{3}$, we have

$$
\begin{aligned}
T_{22}= & \sum_{\substack{b \leq x^{\mu} \\
(b, q)=1}} \sum_{\substack{a \leq x^{\lambda_{2}} \\
(a, q)=1}} \sum_{\substack{c^{5} \leq \frac{x}{a^{3} b^{4}} \\
c^{5} \equiv l \overline{a^{3} b^{4}}(\bmod q)}} 1, \\
= & \frac{x^{(1 / 5)}}{q} \sum_{b \leq x^{\mu}} \frac{1}{b^{(4 / 5)}} \sum_{a \leq x^{\lambda_{2}}} \frac{N_{5}\left(l a^{2} b ; q\right)}{a^{(3 / 5)}}+O\left(x^{\mu+\lambda_{2}} q^{\varepsilon}\right) \\
= & \frac{x^{(1 / 5)}}{q} \sum_{b \leq x^{\mu}} \frac{1}{b^{(4 / 5)}}\left(\sum_{a \leq x^{\lambda_{2}}} \frac{N_{5}\left(l a^{2} b ; q\right)-((\phi(q)) / q)}{a^{(3 / 5)}}+\sum_{a \leq x^{\lambda_{2}}} \frac{1}{a^{(3 / 5)}} \frac{\phi(q)}{q}\right)+O\left(x^{\mu+\lambda_{2}} q^{\varepsilon}\right) \\
= & \frac{x^{(1 / 5)}}{q} \sum_{b \leq x^{\mu}} \frac{1}{b^{(4 / 5)}}\left(\int_{1}^{x^{\lambda_{2}}} \frac{1}{\mu_{(3 / 5)}} \mathrm{d}\left(\sum_{a \leq \mu} N_{5}\left(l a^{2} b ; q\right)-\frac{\phi(q)}{q}\right)\right) \\
& +\frac{x^{(1 / 5)}}{q} \sum_{b \leq x^{\mu}} \frac{1}{b^{(4 / 5)}} \frac{\phi(q)}{q}\left(\frac{5}{2} x^{(2 / 5) \lambda_{2}}-\frac{3}{2}-\frac{3}{5} \int_{1}^{\infty} \frac{t-[t]}{t^{(8 / 5)}} \mathrm{d} t+O\left(x^{-(3 / 5) \lambda_{2}}\right)\right)+O\left(x^{\mu+\lambda_{2}} q^{\varepsilon}\right) .
\end{aligned}
$$

If we let $F_{b}(\mu)=\sum_{a \leq \mu} N_{5}\left(l a^{2} b ; q\right)-((\phi(q)) / q) \mu$, then the first term in the above formula is 
12

Journal of Mathematics

$$
\begin{aligned}
& \frac{x^{(1 / 5)}}{q} \sum_{b \leq x^{\mu}} \frac{1}{b^{(4 / 5)}}\left(O\left(\frac{q^{(1 / 2)+\varepsilon}}{x^{(3 / 5) \lambda_{2}}}\right)-N_{5}(l b ; q)+\frac{\phi(q)}{q}+\frac{3}{5} \int_{1}^{\infty} \frac{F_{b}(\mu)}{\mu^{(8 / 5)}} \mathrm{d} \mu\right) \\
& =-\frac{x^{(1 / 5)}}{q} \sum_{b \leq x^{\mu}} \frac{N_{5}(l b ; q)-((\phi(q)) / q)}{b^{(4 / 5)}}+\frac{3}{5} \frac{x^{(1 / 5)}}{q} \int_{1}^{\infty} \frac{\sum_{b \leq x^{\mu}}\left(F_{b}(\mu) / b^{(4 / 5)}\right)}{\mu^{(8 / 5)}}+O\left(\frac{x^{1 / 5-3 / 5 \lambda_{2}}}{q^{1 / 2-\varepsilon}} \sum_{b \leq x^{\mu}} \frac{1}{b^{4 / 5}}\right) \\
& =\left(N_{5}(l ; q)-\frac{\phi(q)}{q}-\frac{4}{5} \int_{1}^{\infty} \frac{\sum_{b \leq \mu} N_{5}(l b ; q)-((\phi(q)) / q)}{\mu^{(9 / 5)}} d \mu\right) \frac{x^{(1 / 5)}}{q} \\
& \quad+\frac{12}{25} \int_{1}^{\infty} \int_{1}^{\infty} \frac{\sum_{b \leq \lambda} F_{b}(\mu)}{\mu^{(8 / 5)} \lambda^{(9 / 5)}} \mathrm{d} \lambda \mathrm{d} \mu \frac{x^{(1 / 5)}}{q} \\
& \quad-\frac{3}{5} \frac{x^{(1 / 5)}}{q} \int_{1}^{\infty} \frac{\sum_{a \leq \mu} N_{5}\left(l a^{2} ; q\right)-((\phi(q)) / q)}{\mu^{(8 / 5)}} \mathrm{d} \mu \\
& \\
& +O\left(\frac{x^{(1 / 5)-(3 / 5) \lambda_{2}}}{q^{(1 / 2)-\varepsilon}} \sum_{b \leq x^{\mu}} \frac{1}{b^{(4 / 5)}}\right)+O\left(\frac{x^{(1 / 5)-(4 / 5) \mu}}{q^{(1 / 2)-\varepsilon}}\right),
\end{aligned}
$$

and the second term is

$$
\begin{aligned}
\frac{x^{(1 / 5)}}{q} & \sum_{b \leq x^{\mu}} \frac{1}{b^{(4 / 5)}} \frac{\phi(q)}{q} \frac{5}{2} x^{(2 / 5) \lambda_{2}}+\frac{x^{(1 / 5)}}{q} \sum_{b \leq x^{\mu}} \frac{1}{b^{(4 / 5)}} \frac{\phi(q)}{q}\left(-\frac{3}{2}-\frac{3}{5} \int_{1}^{\infty} \frac{t-[t]}{t^{(8 / 5)}} \mathrm{d} t\right)+O\left(\frac{\phi(q)}{q} x^{(1 / 5)-(3 / 5) \lambda_{2}} \sum_{b \leq x^{\mu}} \frac{1}{b^{(4 / 5)}}\right) \\
= & \frac{5}{2} \frac{x^{(1 / 5)+(2 / 5) \lambda}}{q} \sum_{b \leq x^{\mu}} \frac{1}{b^{(4 / 5)}} \frac{\phi(q)}{q}-\frac{x^{(1 / 5)}}{q} \frac{\phi(q)}{q}\left(5 x^{(1 / 5) \mu}-4-\frac{4}{5} \int_{1}^{\infty} \frac{t-[t]}{t^{(9 / 5)}} \mathrm{d} t+O\left(x^{-(4 / 5) \mu}\right)\right)\left(\frac{3}{2}+\frac{3}{5} \int_{1}^{\infty} \frac{t-[t]}{t^{(8 / 5)}} \mathrm{d} t\right) \\
& +O\left(\frac{\phi(q)}{q} x^{(1 / 5)-(3 / 5) \lambda_{2}} \sum_{b \leq x^{\mu}} \frac{1}{b^{(4 / 5)}}\right) \\
= & \frac{5}{2} \frac{\phi(q)}{q^{2}} x^{(1 / 5)+(2 / 5) \lambda_{2}} \sum_{b \leq x^{\mu}} \frac{1}{b^{(4 / 5)}}+\left(-4-\frac{4}{5} \int_{1}^{\infty} \frac{t-[t]}{t^{(9 / 5)}} \mathrm{d} t\right)\left(-\frac{3}{2}-\frac{3}{5} \int_{1}^{\infty} \frac{t-[t]}{t^{(8 / 5)}} \mathrm{d} t\right) \frac{\phi(q)}{q^{2}} x^{(1 / 5)} \\
& +\left(-\frac{15}{2}-3 \int_{1}^{\infty} \frac{t-[t]}{t^{(8 / 5)}} \mathrm{d} t\right) \frac{\phi(q)}{q^{2}} x^{(1 / 5)+(1 / 5) \mu}+O\left(\frac{\phi(q)}{q} x^{(1 / 5)-(3 / 5) \lambda_{2}} \sum_{b \leq x^{\mu}} \frac{1}{b^{(4 / 5)}}\right)+O\left(\frac{\phi(q)}{q^{2}} x^{(1 / 5)-(4 / 5) \mu}\right) .
\end{aligned}
$$

Then, we can obtain

$$
\begin{aligned}
T_{22}= & \frac{x^{(1 / 5)}}{q}\left[\left(-4-\frac{4}{5} \int_{1}^{\infty} \frac{t-[t]}{t^{(9 / 5)}} \mathrm{d} t\right)\left(-\frac{3}{2}-\frac{3}{5} \int_{1}^{\infty} \frac{t-[t]}{t^{(8 / 5)}} \mathrm{d} t\right) \frac{\phi(q)}{q}-\frac{4}{5} \int_{1}^{\infty} \frac{\sum_{b \leq \mu} N_{5}(l b ; q)-((\phi(q)) / q) \mu}{\mu^{(8 / 5)}}\right. \\
& \left.-\frac{3}{5} \frac{x^{(1 / 5)}}{q} \int_{1}^{\infty} \frac{\sum_{a \leq \mu} N_{5}\left(l a^{2} ; q\right)-((\phi(q)) / q)}{\mu^{(8 / 5)}} \mathrm{d} \mu+N_{5}(l ; q)-\frac{\phi(q)}{q}+\frac{12}{25} \int_{1}^{\infty} \int_{1}^{\infty} \frac{\sum_{b \leq \lambda} F_{b}(\mu)}{\mu^{(8 / 5)} \lambda^{(9 / 5)}} \mathrm{d} \lambda \mathrm{d} \mu\right] \\
& +\frac{5}{2} \frac{\phi(q)}{q^{2}} x^{(1 / 5)+(2 / 5) \lambda_{2}} \sum_{b \leq x^{\mu}} \frac{1}{b^{(4 / 5)}}+\left(-\frac{15}{2}-3 \int_{1}^{\infty} \frac{t-[t]}{t^{(8 / 5)}} \mathrm{d} t\right) \frac{\phi(q)}{q^{2}} x^{(1 / 5)+(1 / 5) \mu} \\
& +O\left(\frac{x^{(1 / 5)-(3 / 5) \lambda_{2}}}{q^{(1 / 2)-\varepsilon}} \sum_{b \leq x^{\mu}} \frac{1}{b^{(4 / 5)}}+\frac{x^{(1 / 5)-(4 / 5) \mu}}{q^{(1 / 2)-\varepsilon}}+\frac{\phi(q)}{q^{2}} x^{(1 / 5)-(4 / 5) \mu}+x^{\mu+\lambda_{2}} q^{\varepsilon}\right) .
\end{aligned}
$$


If we pick $\lambda_{1}$ to have the same size as $\mu_{1}$, we can get

$$
\begin{aligned}
T_{2}= & \sum_{b \leq x^{\mu}} \frac{1}{b^{(4 / 3)}}\left(\sum_{c=1}^{\infty} \frac{N_{3}\left(l b^{2} c ; q\right)}{c^{(5 / 3)}}\right) \frac{x^{(1 / 3)}}{q}+\frac{x^{(1 / 5)}}{q}\left[\left(-4-\frac{4}{5} \int_{1}^{\infty} \frac{t-[t]}{t^{(9 / 5)}} \mathrm{d} t\right)\left(-\frac{3}{2}-\frac{3}{5} \int_{1}^{\infty} \frac{t-[t]}{t^{(8 / 5)}} \mathrm{d} t\right) \frac{\phi(q)}{q}\right. \\
& -\frac{4}{5} \int_{1}^{\infty} \frac{\sum_{b \leq \mu} N_{5}(l b ; q)-((\phi(q)) / q) \mu}{\mu^{(8 / 5)}} \mathrm{d} \mu+N_{5}(l ; q)-\frac{\phi(q)}{q}-\frac{3}{5} \frac{x^{(1 / 5)}}{q} \int_{1}^{\infty} \frac{\sum_{a \leq \mu} N_{5}\left(l a^{2} ; q\right)-((\phi(q)) / q)}{\mu^{(8 / 5)}} \mathrm{d} \mu \\
& \left.+\frac{12}{25} \int_{1}^{\infty} \int_{1}^{\infty} \frac{\sum_{b \leq \lambda} F_{b}(\mu)}{\mu^{(8 / 5)} \lambda^{(9 / 5)}} \mathrm{d} \lambda \mathrm{d} \mu\right]+\left(-\frac{15}{2}-3 \int_{1}^{\infty} \frac{t-[t]}{t^{(8 / 5)}} \mathrm{d} t\right) \frac{\phi(q)}{q^{2}} x^{(1 / 5)+(1 / 5) \mu} \\
& +O\left(\frac{x^{(1 / 3)-(5 / 3) \mu_{2}}}{q^{(1 / 2)-\varepsilon}}+\frac{x^{(1 / 5)-(3 / 5) \lambda_{2}}}{q^{(1 / 2)-\varepsilon}} \sum_{b \leq x^{\mu}} \frac{1}{b^{(4 / 5)}}+\frac{x^{(1 / 5)-(4 / 5) \mu}}{q^{(1 / 2)-\varepsilon}}+x^{\mu+\mu_{2}} q^{\varepsilon}+2^{J_{2}} q^{(1 / 2)+\varepsilon} x^{\mu}\right)
\end{aligned}
$$

where $2^{J_{2}}$ satisfies $\left(X^{\mu_{1}} / 2^{J_{2}} q^{(1 / 2)}\right) \ll 1$.

Similar to the proofs of $T_{2}$ and $T_{3}$, we can get the following:

$$
\begin{aligned}
& T_{1}=\sum_{a \leq x^{\lambda}} \frac{1}{a^{(3 / 4)}}\left(\sum_{c=1}^{\infty} \frac{N_{4}\left(l a c^{3} ; q\right)}{c^{(5 / 4)}}\right) \frac{x^{(1 / 4)}}{q} \\
& +\sum_{a \leq x^{\lambda}} \frac{1}{a^{(3 / 5)}}\left(-4 \frac{\phi(q)}{q}-N_{5}\left(l a^{2} ; q\right)+\frac{4}{5} \int_{1}^{\infty} \frac{\sum_{l_{1} \leq \mu} N_{5}\left(l l_{1} a^{2} ; q\right)-((\phi(q)) / q) \mu}{\mu^{(9 / 5)}} \mathrm{d} \mu\right) \frac{x^{(1 / 5)}}{q} \\
& +O\left(\frac{x^{(1 / 4)-(5 / 4) \mu_{3}}}{q^{(1 / 2)-\varepsilon}} \sum_{a \leq x^{\lambda}} \frac{1}{a^{(3 / 4)}}+x^{\lambda+\mu_{3}} q^{\varepsilon}+2^{J_{3}} q^{(1 / 2)+\varepsilon} x^{\lambda}+\frac{x^{(1 / 5)-(4 / 5) \lambda_{3}}}{q^{(1 / 2)-\varepsilon}} \sum_{a \leq x^{\lambda}} \frac{1}{a^{(3 / 5)}}\right), \\
& T_{4}=\left(\frac{5}{2} x^{(2 / 5) \lambda}-\frac{3}{2}-\frac{3}{5} \int_{1}^{\infty} \frac{t-[t]}{t^{(8 / 5)}} \mathrm{d} t+O\left(x^{-(3 / 5) \lambda}\right)\right) \frac{5 \phi(q)}{q^{2}} x^{(1 / 5)+(1 / 5) \mu}+O\left(\frac{x^{(1 / 5)-(4 / 5) \mu}}{q^{(1 / 2)-\varepsilon}} \sum_{a \leq x^{\lambda}} \frac{1}{a^{(3 / 5)}}\right) \\
& +\sum_{a \leq x^{\lambda}} \frac{1}{a^{(3 / 5)}}\left(-4 \frac{\phi(q)}{q}-N_{5}\left(l a^{2} ; q\right)+\frac{4}{5} \int_{1}^{\infty} \frac{\sum_{l_{1} \leq \mu} N_{5}\left(l l_{1} a^{2} ; q\right)-((\phi(q)) / q) \mu}{\mu^{(9 / 5)}} d \mu\right) \frac{x^{(1 / 5)}}{q} \\
& =\frac{25}{2} \frac{\phi(q)}{q^{2}} x^{(1 / 5)+(\mu / 5)+(2 / 5) \lambda}+\left(-\frac{15}{2}-3 \int_{1}^{\infty} \frac{t-[t]}{t^{(8 / 5)}} \mathrm{d} t\right) \frac{\phi(q)}{q^{2}} x^{(1 / 5)+(1 / 5) \mu} \\
& +\sum_{a \leq x^{\lambda}} \frac{1}{a^{(3 / 5)}}\left(-4 \frac{\phi(q)}{q}-N_{5}\left(l a^{2} ; q\right)+\frac{4}{5} \int_{1}^{\infty} \frac{\sum_{l_{1} \leq \mu} N_{5}\left(l l_{1} a^{2} ; q\right)-((\phi(q)) / q) \mu}{\mu^{(9 / 5)}} \mathrm{d} \mu\right) \frac{x^{(1 / 5)}}{q} \\
& +O\left(\frac{\phi(q)}{q^{2}} x^{(1 / 5)+(\mu / 5)-(3 / 5) \lambda}\right)+O\left(\frac{x^{(1 / 5)-(4 / 5) \mu}}{q^{(1 / 2)-\varepsilon}} \sum_{a \leq x^{\lambda}} \frac{1}{a^{(3 / 5)}}\right)
\end{aligned}
$$




$$
\begin{aligned}
& T_{5}=\sum_{b \leq x^{\mu}} \frac{1}{b^{(4 / 3)}}\left(\sum_{c=1}^{\infty} \frac{N_{3}\left(l b^{2} c ; q\right)}{c^{(5 / 3)}}\right) \frac{x^{(1 / 3)}}{q}-\frac{3}{2} \frac{\phi(q)}{q^{2}} x^{(1 / 3)-(2 / 3) v} \sum_{b \leq x^{\mu}} \frac{1}{b^{4 / 3}}+O\left(\frac{x^{(1 / 3)-(2 / 3) \mu}}{q^{(1 / 2)-\varepsilon}} \sum_{b \leq x^{\mu}} \frac{1}{b^{(4 / 3)}}\right) \\
& =\sum_{b \leq x^{\mu}} \frac{1}{b^{(4 / 3)}}\left(\sum_{c=1}^{\infty} \frac{N_{3}\left(l b^{2} c ; q\right)}{c^{(5 / 3)}}\right) \frac{x^{(1 / 3)}}{q}+\frac{3}{2} \frac{\phi(q)}{q^{2}} x^{(1 / 3)-(2 / 3) v}\left(3 x^{-(1 / 3) \mu}-4+\frac{4}{3} \int_{1}^{\infty} \frac{t-[t]}{t^{(7 / 3)}} \mathrm{d} t+O\left(x^{-(4 / 3) \mu}\right)\right) \\
& +O\left(\frac{x^{(1 / 3)-(5 / 3) \mu}}{q^{(1 / 2)-\varepsilon}} \sum_{b \leq x^{\mu}} \frac{1}{b^{(4 / 3)}}\right) \\
& =\sum_{b \leq x^{\mu}} \frac{1}{b^{(4 / 3)}}\left(\sum_{c=1}^{\infty} \frac{N_{3}\left(l b^{2} c ; q\right)}{c^{(5 / 3)}}\right) \frac{x^{(1 / 3)}}{q}+\frac{9}{2} \frac{\phi(q)}{q^{2}} x^{(1 / 3)-(2 / 3) v-(1 / 3) \mu}+\left(-6+2 \int_{1}^{\infty} \frac{t-[t]}{t^{(7 / 3)}} \mathrm{d} t\right) \frac{\phi(q)}{q} x^{(1 / 3)-(2 / 3) v} \\
& +O\left(\frac{\phi(q)}{q^{2}} x^{(1 / 3)-(2 / 3) \nu-(4 / 3) \mu}\right)+O\left(\frac{x^{(1 / 3)-(5 / 3) \mu}}{q^{(1 / 2)-\varepsilon}} \sum_{b \leq x^{\mu}} \frac{1}{b^{(4 / 3)}}\right) \\
& T_{6}=\sum_{a \leq x^{\lambda}} \frac{1}{a^{(3 / 4)}}\left(\sum_{c=1}^{\infty} \frac{N_{4}\left(l a c^{3} ; q\right)}{c^{(5 / 4)}}\right) \frac{x^{(1 / 4)}}{q}-\frac{4 \phi(q)}{q^{2}} x^{(1 / 4)-(1 / 4) v} \sum_{a \leq x^{\lambda}} \frac{1}{a^{(3 / 4)}}+O\left(\frac{x^{(1 / 4)-(5 / 4) v}}{q^{(1 / 2)-\varepsilon}} \sum_{a \leq x^{\lambda}} \frac{1}{a^{(3 / 4)}}\right) \\
& =\sum_{a \leq x^{\lambda}} \frac{1}{a^{(3 / 4)}}\left(\sum_{c=1}^{\infty} \frac{N_{4}\left(l a c^{3} ; q\right)}{c^{(5 / 4)}}\right) \frac{x^{(1 / 4)}}{q}-\frac{4 \phi(q)}{q^{2}} x^{(1 / 4)-(21 / 4) v}\left(4 x^{(1 / 4) \lambda}-3-\frac{3}{4} \int_{1}^{\infty} \frac{t-[t]}{t^{(7 / 4)}} \mathrm{d} t+O\left(x^{-(3 / 4) \lambda}\right)\right) \\
& +O\left(\frac{x^{(1 / 4)-(5 / 4) v}}{q^{(1 / 2)-\varepsilon}} \sum_{a \leq x^{\lambda}} \frac{1}{a^{(3 / 4)}}\right) \\
& =\sum_{a \leq x^{\lambda}} \frac{1}{a^{(3 / 4)}}\left(\sum_{c=1}^{\infty} \frac{N_{4}\left(l a c^{3} ; q\right)}{c^{(5 / 4)}}\right) \frac{x^{(1 / 4)}}{q}-16 \frac{\phi(q)}{q^{2}} x^{(1 / 4)-(1 / 4) v+(1 / 4) \lambda}+\left(12+3 \int_{1}^{\infty} \frac{t-[t]}{t^{(7 / 4)}} \mathrm{d} t\right) \frac{\phi(q)}{q} x^{(1 / 4)-(1 / 4) v} \\
& +O\left(\frac{\phi(q)}{q^{2}} x^{(1 / 4)-(1 / 4) v-(1 / 4) \lambda}\right)+O\left(\frac{x^{(1 / 4)-(5 / 4) v}}{q^{(1 / 2)-\varepsilon}} \sum_{a \leq x^{\lambda}} \frac{1}{b^{(3 / 4)}}\right) \\
& T_{7}=\sum_{\substack{a \leq x^{\lambda} \\
(a, q)=1}} \sum_{\substack{b \leq x^{\mu} \\
(b, q)=1}} \sum_{\substack{c \leq x^{\nu} \\
(c, q)=1 \\
a^{3} b^{4} c^{5} \equiv l(\bmod q)}} 1=\frac{\phi(q)}{q} x^{\lambda+\mu+\nu}+O\left(\frac{x^{\lambda}}{q^{(1 / 2)-\varepsilon}} x^{\mu+\nu}\right) .
\end{aligned}
$$

Finally, we discuss $T_{8}$ as follows: dividing interval $\left[x^{\nu}, x^{((1-3 \lambda-4 \mu) / 5)}\right]$ into $m$ parts with the length of each one being $\left(\left(x^{((1-3 \lambda-4 \mu) / 5)}-x^{\nu}\right) / m\right)$. The $n$th part is

$$
\left[x^{\nu}+(n-1) \frac{x^{((1-3 \lambda-4 \mu) / 5)}-x^{\nu}}{m}, x^{\nu}+n \frac{x^{((1-3 \lambda-4 \mu) / 5)}-x^{\nu}}{m}\right] \text {. }
$$

Let $a_{0}$ be the intersection of the plane $b=x^{\mu}$, $c=x^{\nu}+n\left(\left(x^{((1-3 \lambda-4 \mu) / 5)}-x^{\nu}\right) / m\right)$, and curved surface $a^{3} b^{4} c^{5}=x$, which is

$$
a_{0}^{3} x^{4 \mu}\left(x^{\nu}+n \frac{x^{((1-3 \lambda-4 \mu) / 5)}-x^{\nu}}{m}\right)^{5}=x,
$$

and then we obtain

$$
a_{0}=\frac{x^{((1-4 \mu) / 3)}}{\left(x^{\nu}+n\left(\left(x^{((1-3 \lambda-4 \mu) / 5)}-x^{\nu}\right) / m\right)\right)^{(5 / 3)}} .
$$

Similarly, we have

$$
b_{0}=\frac{x^{((1-3 \lambda) / 4)}}{\left(x^{\nu}+n\left(\left(x^{((1-3 \lambda-4 \mu) / 5)}-x^{\nu}\right) / m\right)\right)^{(5 / 4)}}
$$

We define the intersection of $a^{3} b^{4} c^{5}=x$ and $x^{\nu}+$ $n\left(\left(x^{((1-3 \lambda-4 \mu) / 5)}-x^{\nu}\right) / m\right)$ as 


$$
a^{3} b^{4}=\frac{x}{\left(x^{\nu}+n\left(\left(x^{((1-3 \lambda-4 \mu) / 5)}-x^{\nu}\right) / m\right)\right)^{5}}:=x_{n} .
$$

Now we divide the interval in the $n$th part and follow the construction in [23]; firstly, we have rectangles

$$
R_{i}=\left[2^{i-1} x^{\lambda}, 2^{i} x^{\lambda}\right] \times\left[x^{\mu},\left(\frac{x_{n}}{\left(2^{i} x^{\lambda}\right)^{3}}\right)^{(1 / 4)}\right], 1 \leq i \leq I=\log _{2}\left(\frac{a_{0}}{x^{\lambda}}\right) .
$$

In the remaining regions

$$
S_{i}:=\left\{(a, b), 2^{i-1} x^{\lambda}<a \leq 2^{i} x^{\lambda}, b>\left(\frac{x_{n}}{\left(2^{i} x^{\lambda}\right)^{3}}\right)^{(1 / 4)}, a^{3} b^{4} \leq x^{m}\right\}
$$

we place additional rectangles $R_{i j k}$. If we let $1 \leq k \leq 2^{j-1}$, in the same way we place a further rectangles and so on, then we can get $R_{i j k}$ which is

$$
R_{i j k}=\left(2^{i-1}\left(1+\frac{2 k-2}{2^{j}}\right) x^{\lambda}, 2^{i-1}\left(1+\frac{2 k-1}{2^{j}}\right) x^{\lambda}\right] \times\left(\left(\frac{x_{n}}{\left(2^{i-1}\left(1+\left(2 k / 2^{j}\right)\right) x^{\lambda}\right)^{3}}\right)^{(1 / 4)},\left(\frac{x_{n}}{\left(2^{i-1}\left(1+\left(2 k-1 / 2^{j}\right)\right) x^{\lambda}\right)^{3}}\right)^{(1 / 4)}\right]
$$

The remaining regions are

$$
\begin{aligned}
S_{i j k} & =\left\{(a, b): 2^{i-1}\left(1+\frac{2 k-2}{2^{j}}\right) x^{\lambda}<a \leq 2^{i-1}\left(1+\frac{2 k-1}{2^{j}}\right) x^{\lambda},\right. \\
b & \left.>\left(\frac{x_{n}}{\left(2^{i-1}\left(1+\left((2 k-1) / 2^{j}\right)\right) x^{\lambda}\right)^{3}}\right)^{(1 / 4)}, a^{3} b^{4} \leq x_{n}\right\}, \\
S_{i j k}^{\prime} & =\left\{(a, b): 2^{i-1}\left(1+\frac{2 k-1}{2^{j}}\right) x^{\lambda}<a \leq 2^{i-1}\left(1+\frac{2 k}{2^{j}}\right) x^{\lambda},\right. \\
b & \left.>\left(\frac{x_{n}}{\left(2^{i-1}\left(1+\left(2 k / 2^{j}\right)\right) x^{\lambda}\right)^{3}}\right)^{(1 / 4)}, a^{3} b^{4} \leq x_{n}\right\} .
\end{aligned}
$$

In this part, expanding $R_{i}$ and $R_{i j k}$ to a cube with height $\left(\left(x^{((1-3 \lambda-4 \mu) / 5)}-x^{\nu}\right) / m\right)$, we can get $R_{n i}, R_{n i j k}, S_{n i}, S_{n i j k} S_{n i j k}^{\prime}$, and the remaining region $S_{n}$ correspondingly. Then, we have the asymptotic formula

$$
\begin{aligned}
T_{8}= & \sum_{n} \sum_{i=1}^{I-1} \sum_{\substack{(a, b, c) \in R_{n i} \\
a^{3} b^{4} c^{5} \equiv l(\bmod q)}} 1+\sum_{n} \sum_{j=1}^{J} \sum_{i=1}^{I} \sum_{k=1}^{2^{j-1}} \sum_{\substack{(a, b, c) \in R_{n j i k} \\
a^{3} b^{4} c^{5} \equiv l(\bmod q)}} 1+\sum_{n} \sum_{i=1}^{I} \sum_{k=1}^{2^{J-1}} \sum_{\substack{(a, b, c) \in S_{n j i k} \\
a^{3} b^{4} c^{5} \equiv l(\bmod q)}} 1 \\
& +\sum_{n} \sum_{i=1}^{I} \sum_{k=1}^{2^{J-1}} \sum_{\substack{(a, b, c) \in S_{n i j k}^{\prime} \\
a^{3} b^{4} c^{5} \equiv l(\bmod q)}} 1+\sum_{n} \sum_{\substack{(a, b, c) \in S_{n} \\
a^{3} b^{4} c^{5} \equiv l(\bmod q)}} 1 .
\end{aligned}
$$

If we let $N_{A, B, C}(K, L, M):=\#\{(a, b, c)$ : $a^{3} b^{4} c^{5} \equiv l(\bmod q), A<a \leq A+K, B<b \leq B+L, C<c \leq C+$ $M$ \}, by Lemma 5 , we know

$$
N_{A, B, C}(K, L, M)=\frac{\phi(q)}{q^{2}} K L M+O\left(\frac{M}{q^{(1 / 2)-\varepsilon}}+K L q^{\varepsilon}\right) .
$$


Then, we further obtain

$$
\begin{aligned}
& T_{8}=\frac{\phi(q)}{q^{2}} \text { Area of }\left\{(a, b, c): a>x^{\lambda}, b>x^{\mu}, c>x^{\nu}, a^{3} b^{4} c^{5} \leq x\right\} \\
& +O\left(\sum_{n} \sum_{i=1}^{I_{n}} \sum_{k=1}^{J^{J-1}} \frac{\text { Area of } S_{n i J k}}{q}\right)+O\left(\sum_{n} \sum_{i=1}^{I_{n}} \sum_{k=1}^{2^{J-1}} \frac{\text { Area of } S_{n i J k}^{\prime}}{q}\right) \\
& +O\left(\sum_{n} \sum_{i=1}^{I} \sum_{k=1}^{2^{J-1}} \sum_{\begin{array}{c}
(a, b, c) \in S_{n i j k} \\
a^{3} b^{4} c^{5} \equiv l(\bmod q)
\end{array}} 1\right)+O\left(\sum_{n} \sum_{i=1}^{I} \sum_{k=1}^{2^{J-1}} \sum_{\begin{array}{c}
(a, b, c) \in S_{n i j k}^{\prime} \\
a^{3} b^{4} c^{5} \equiv l(\bmod q)
\end{array}} 1\right) \\
& +O\left(\sum_{n} \sum_{i=1}^{I_{n}} \frac{1}{q^{(1 / 2)-\varepsilon}} \frac{x^{((1-3 \lambda-4 \mu) / 5)}-x^{\nu}}{m}+\sum_{n} \sum_{i=1}^{I_{n}} 2^{i-1} x^{\lambda}\left(\frac{x_{n}^{(1 / 4)}}{\left(2^{i} x^{\lambda}\right)^{(3 / 4)}}-x^{\mu}\right)\right. \\
& \left.+\sum_{n} \sum_{j=1}^{J} \sum_{i=1}^{I_{n}} \sum_{k=1}^{j-1} \frac{x^{((1-3 \lambda-4 \mu) / 5)}-x^{\nu}}{m} \frac{1}{q^{(1 / 2)}}+\sum_{n} \sum_{j=1}^{J} \sum_{i=1}^{I_{n}} \sum_{k=1}^{j^{j-1}} 2^{i-1} x^{\lambda} \frac{1}{2^{2 j}} \frac{x_{n}^{(1 / 4)}}{\left(2^{i-1} x^{\lambda}\right)^{(1 / 4)}}\right)+O\left(\sum_{n} \sum_{\substack{(a, b, c) \in S_{n} \\
a^{3} b^{4} c^{5} \equiv l(\bmod q)}} 1\right),
\end{aligned}
$$

in which the main term is

$$
\begin{aligned}
& \frac{\phi(q)}{q^{2}} \int_{x^{\nu}}^{x^{((1-3 \lambda-4 \mu / 5)}} \int_{x^{\lambda}}^{\left(x^{((1-4 \mu) / 3)} / c^{(5 / 3)}\right)}\left(\left(\frac{x}{a^{3} c^{5}}\right)^{(1 / 4)}-x^{\mu}\right) \mathrm{d} a \mathrm{~d} c \\
& =\frac{\phi(q)}{q^{2}}\left(\frac{25}{2} x^{((1+2 \lambda+\mu) / 5)}+\frac{9}{2} x^{((1-\mu-2 \nu) / 3)}-16 x^{((1+\lambda-\nu) / 4)}-x^{\lambda+\mu+\nu}\right) .
\end{aligned}
$$

Now we deal with the error term of $T_{8}$. Note that

$$
\begin{aligned}
S_{n i j k} \subseteq & \left.2^{i-1}\left(1+\frac{2 k-2}{2^{J}}\right) x^{\lambda}, 2^{i-1}\left(1+\frac{2 k-1}{2^{J}}\right) x^{\lambda}\right] \\
& \times\left[\left(\frac{x_{n}}{\left(2^{i-1}\left(1+\left((2 k-1) / 2^{j}\right)\right) x^{\lambda}\right)^{3}}\right)^{(1 / 4)},\left(\frac{x_{n}}{\left(2^{i-1}\left(1+\left((2 k-2) / 2^{j}\right)\right) x^{\lambda}\right)^{3}}\right)^{(1 / 4)}\right] \\
& \times\left[x^{\nu}+(n-1) \frac{x^{((1-3 \lambda-4 \mu) / 5)}-x^{\nu}}{m}, x^{\nu}+n \frac{x^{((1-3 \lambda-4 \mu) / 5)}-x^{\nu}}{m}\right] .
\end{aligned}
$$

Thus, the area of $S_{n i J k}$ is at most

$$
\frac{2^{i-1} x^{\lambda}}{2^{J}} \cdot \frac{x_{n}^{(1 / 4)}}{\left(2^{i-1} x^{\lambda}\right)^{(3 / 4)}} \cdot \frac{1}{2^{J}} \cdot \frac{x^{((1-3 \lambda-4 \mu) / 5)}-x^{\nu}}{m},
$$

which implies that the estimation of the first error term is

$$
\sum_{n} \sum_{i=1}^{I_{n}} \sum_{k=1}^{2^{J-1}} \frac{\text { Area of } S_{n i J k}}{q} \ll \frac{1}{2^{J}} \cdot \frac{1}{q} \cdot \frac{m^{(2 / 3)} x^{((1-\mu) / 3)}}{\left(x^{((1-3 \lambda-4 \mu) / 5)}\right)^{(2 / 3)}} .
$$


Similarly, we have the same error bound for the second, third, and the fourth error term. The error terms in the fifth are

$$
\begin{aligned}
& \sum_{n} \sum_{i=1}^{I_{n}} \frac{1}{q^{(1 / 2)-\varepsilon}} \frac{x^{((1-3 \lambda-4 \mu) / 5)}-x^{\nu}}{m} \ll \frac{1}{q^{(1 / 2)}}\left(x^{((1-3 \lambda-4 \mu) / 5)}-x^{\nu}\right), \\
& \sum_{n} \sum_{i=1}^{I_{n}} 2^{i-1} x^{\lambda}\left(\frac{x_{n}^{(1 / 4)}}{\left(2^{i} x^{\lambda}\right)^{(3 / 4)}}-x^{\mu}\right) \ll \frac{m^{(5 / 3)} x^{((1-\mu) / 3)}}{\left(x^{((1-3 \lambda-4 \mu) / 5)}\right)^{(5 / 3)}}, \\
& \sum_{n} \sum_{j=1}^{J} \sum_{i=1}^{I_{n}} \sum_{k=1}^{2^{j-1}} \frac{x^{((1-3 \lambda-4 \mu) / 5)}-x^{\nu}}{m} \frac{1}{q^{(1 / 2)}} \ll 2^{J} \frac{1}{q^{(1 / 2)}}\left(x^{((1-3 \lambda-4 \mu) / 5)}-x^{\nu}\right), \\
& \sum_{n} \sum_{j=1}^{J} \sum_{i=1}^{I_{n}} \sum_{k=1}^{2^{j-1}} 2^{i-1} x^{\lambda} \frac{1}{2^{2 j}} \frac{x_{n}^{(1 / 4)}}{\left(2^{i-1} x^{\lambda}\right)^{(1 / 4)}} \ll \frac{m^{(5 / 3)} x^{((1-\mu) / 3)}}{\left(x^{((1-3 \lambda-4 \mu) / 5)}\right)^{(5 / 3)}} .
\end{aligned}
$$

For the last error term, we extend $S_{n}$ into a ring sector which volume is

$$
\frac{x^{((1-3 \lambda-4 \mu) / 5)}-x^{\nu}}{m}\left(\int_{x^{\lambda}}^{a_{1}}\left[\left(\frac{x_{n-1}}{a^{3}}\right)^{(1 / 4)}-x^{\mu}\right] \mathrm{d} a-\int_{x^{\lambda}}^{a_{0}}\left[\left(\frac{x_{n}}{a^{3}}\right)^{(1 / 4)}-x^{\mu}\right] \mathrm{d} a\right),
$$

where

$$
a_{1}^{3} x^{4 \mu}\left(x^{\nu}+(n-1) \frac{x^{((1-3 \lambda-4 \mu) / 5)}-x^{\nu}}{m}\right)^{5}=x .
$$

$$
\sum_{n} \sum_{\substack{(a, b, c) \in S_{n} \\ a^{3} b^{4} c^{c} \equiv l(\bmod q)}} 1 \ll \frac{1}{m} x^{((1-3 \lambda-4 \mu) / 5)} x^{((1-\mu-5 v) / 3)}=\frac{1}{m} x^{((8-9 \lambda-17 \mu-25 v) / 15)} .
$$

Then, we can add $n$ and get

Therefore, we have

$$
\begin{aligned}
T_{8}= & \frac{\phi(q)}{q^{2}}\left(\frac{25}{2} x^{((1+2 \lambda+\mu) / 5)}+\frac{9}{2} x^{((1-\mu-2 v) / 3)}-16 x^{((1+\lambda-\nu) / 4)}-x^{\lambda+\mu+\nu}\right) \\
& +O\left(\frac{1}{2^{J}} \cdot \frac{1}{q} \cdot \frac{m^{(2 / 3)} x^{((1-\mu) / 3)}}{\left(x^{((1-3 \lambda-4 \mu) / 5)}\right)^{(2 / 3)}}+\frac{1}{q^{(1 / 2)}}\left(x^{((1-3 \lambda-4 \mu) / 5)}-x^{\nu}\right)+\frac{m^{(5 / 3)} x^{((1-\mu) / 3)}}{\left(x^{((1-3 \lambda-4 \mu) / 5)}\right)^{(5 / 3)}}\right. \\
& \left.+2^{J} \frac{1}{q^{(1 / 2)}}\left(x^{((1-3 \lambda-4 \mu) / 5)}-x^{\nu}\right)+\frac{1}{m} x^{((8-9 \lambda-17 \mu-25 \nu) / 15)}\right) .
\end{aligned}
$$

Now we come to simplify the above error terms. Firstly, we have

$$
\frac{1}{q^{(1 / 2)}}\left(x^{((1-3 \lambda-4 \mu) / 5)}-x^{\nu}\right) \ll \frac{x^{(1 / 5)-(2 / 5) \lambda}}{q^{(1 / 2)-\varepsilon}} .
$$

When $m=O(1)$ and $\lambda=\mu$, we know that

$$
\frac{m^{(5 / 3)} x^{((1-\mu) / 3)}}{\left(x^{((1-3 \lambda-4 \mu) / 5)}\right)^{(5 / 3)}} \ll x^{2 \lambda} .
$$


If $2^{J} \ll x^{\lambda}$ and $\lambda=\mu$, then

$$
2^{J} \frac{1}{q^{(1 / 2)}}\left(x^{((1-3 \lambda-4 \mu) / 5)}-x^{\nu}\right) \ll \frac{x^{(1 / 5)-(2 / 5) \lambda}}{q^{(1 / 2)-\varepsilon}} .
$$

If we pick $2^{J}=x^{\lambda}$ and $\lambda=\mu$, we get

$$
\frac{1}{2^{J}} \cdot \frac{1}{q} \cdot \frac{m^{(2 / 3)} x^{((1-\mu) / 3)}}{\left(x^{((1-3 \lambda-4 \mu) / 5)}\right)^{(2 / 3)}} \ll \frac{x^{(1 / 5)-(2 / 5) \lambda}}{q^{(1 / 2)-\varepsilon}},
$$

with the condition $m=O(1)$.

Therefore, if we choose $\lambda=\mu=\nu, \mu_{i}=\lambda_{i}(i=1,2,3)$ and $m=O(1)$, then we get all error terms from $T_{1}$ to $T_{8}$ :

$$
\begin{aligned}
& O\left(\frac{x^{(1 / 3)-(4 / 3) \mu_{1}}}{q^{(1 / 2)-\varepsilon}}+x^{\mu_{1}+\lambda} q^{\varepsilon}+2^{J_{1}} q^{(1 / 2)+\varepsilon} x^{\lambda}+\frac{x^{(1 / 3)-(5 / 3) \mu_{2}+(1 / 5) \lambda}}{q^{(1 / 2)-\varepsilon}}+x^{\mu_{2}+\lambda} q^{\varepsilon}+2^{J_{2}} q^{(1 / 2)+\varepsilon} x^{\lambda}\right. \\
& \left.+\frac{x^{(1 / 4)-(5 / 4) \mu_{3}+(2 / 5) \lambda}}{q^{(1 / 2)-\varepsilon}}+x^{\mu_{3}+\lambda} q^{\varepsilon}+2^{J_{3}} q^{(1 / 2)+\varepsilon} x^{\lambda}+\frac{x^{(1 / 3)-(5 / 3) \lambda}}{q^{(1 / 2)-\varepsilon}}+\frac{x^{(1 / 5)-(2 / 5) \lambda}}{q^{(1 / 2)-\varepsilon}}+x^{(8 / 15)-(51 / 15) \lambda}\right),
\end{aligned}
$$

where $\mu_{1}, \mu_{2}$, and $\mu_{3}$ satisfy the conditions

Further if we choose $\eta=\mu_{1}=\mu_{2}=\mu_{3}$, then we obtain

$$
\begin{aligned}
\frac{1}{10} & <\mu_{1}<\frac{1}{5}-\lambda, & \frac{x^{\mu_{1}}}{2^{J_{1}} q^{(1 / 2)}} & \ll 1, \\
\frac{2}{25}+\frac{3}{25} \lambda & <\mu_{2}<\frac{1}{5}-\lambda, & \frac{x^{\mu_{2}}}{2^{J_{2}} q^{(1 / 2)}} & \ll 1, \\
\frac{1}{25}+\frac{8}{25} \lambda & <\mu_{3}<\frac{1}{5}-\lambda, & \frac{x^{\mu_{3}}}{2^{J_{3}} q^{(1 / 2)}} & \ll 1 .
\end{aligned}
$$

$$
O\left(\frac{(1 / 3)-(4 / 3) \eta}{q^{(1 / 2)-\varepsilon}}+x^{\eta+\lambda} q^{\varepsilon}+2^{J_{1}} q(1 / 2)^{(1 / 2)+\varepsilon} x^{\lambda}+\frac{x^{(1 / 5)-(2 / 5) \lambda}}{q^{(1 / 2)-\varepsilon}}+x^{(8 / 15)-(51 / 15) \lambda}+\frac{x^{(1 / 3)-(5 / 3) \lambda}}{q^{(1 / 2)-\varepsilon}}\right)
$$

where $\quad\left(x^{\eta} /\left(2^{J_{1}} q^{(1 / 2)}\right)\right) \ll 1, \quad(5 / 51)<\lambda<(1 / 10) \quad$ and $(1 / 10)<\eta<(1 / 5)-\lambda$. Continue to simplify the error term and get

$$
O\left(\frac{x^{(1 / 3)-(4 / 3) \eta}}{q^{(1 / 2)-\varepsilon}}+x^{\eta+\lambda} q^{\varepsilon}+2^{J_{1}} q^{(1 / 2)+\varepsilon} x^{\lambda}\right)
$$

where

$$
\left(x^{\eta} /\left(2^{J_{1}} q^{(1 / 2)}\right)\right) \ll 1
$$

and

$\left\{\begin{array}{l}(8 / 15)-(66 / 15) \lambda<\eta<(1 / 5)-\lambda, \\ (5 / 51)<\lambda<(13 / 132),\end{array}\right.$

or

$$
\sum_{\substack{a^{3} b^{4} c^{5} \leq x \\ a^{3} b^{4} c^{5} \equiv l(\bmod q)}} 1=A_{q}^{\prime}(l) \frac{x^{(1 / 3)}}{q}+B_{q}^{\prime}(l) \frac{x^{(1 / 4)}}{q}+C_{q}^{\prime}(l) \frac{x^{(1 / 5)}}{q}+O\left(\frac{x^{(1 / 3)-(4 / 3) \eta}}{q^{(1 / 2)-\varepsilon}}+\max \left(x^{\eta}, q^{(1 / 2)}\right) q^{\varepsilon} x^{\lambda}\right),
$$


where

$$
\begin{aligned}
A_{q}^{\prime}(l)= & \sum_{b=1}^{\infty} \sum_{c=1}^{\infty} \frac{N_{3}\left(l b^{2} c ; q\right)}{b^{(4 / 3)} c^{(5 / 3)}} \\
B_{q}^{\prime}(l)= & \frac{\phi(q)}{q}\left(5-\frac{5}{4} \int_{1}^{\infty} \frac{t-[t]}{t^{(9 / 4)}} \mathrm{d} t\right)\left(-3-\frac{3}{4} \int_{1}^{\infty} \frac{t-[t]}{t^{(7 / 4)}} \mathrm{d} t\right)-\sum_{c=1}^{\infty} \frac{N_{4}\left(l c^{3} ; q\right)-((\phi(q)) / q)}{c^{(5 / 4)}} \\
& +\frac{3}{4} \frac{x^{(1 / 4)}}{q} \int_{1}^{\infty}\left(\sum_{c=1}^{\infty} \frac{\sum_{a \leq \mu} N_{4}\left(l a c^{3} ; q\right)-((\phi(q)) / q) \mu}{c^{(5 / 4)}}\right) \mu^{-(7 / 4)} \mathrm{d} \mu, \\
C_{q}^{\prime}(l)= & \left(-4-\frac{4}{5} \int_{1}^{\infty} \frac{t-[t]}{t^{(9 / 5)}} \mathrm{d} t\right)\left(-\frac{3}{2}-\frac{3}{5} \int_{1}^{\infty} \frac{t-[t]}{t^{(8 / 5)}} \mathrm{d} t\right) \frac{\phi(q)}{q}-\frac{4}{5} \int_{1}^{\infty} \frac{\sum_{b \leq \mu} N_{5}(l b ; q)-((\phi(q)) / q) \mu}{\mu^{(8 / 5)}} \mathrm{d} \mu \\
& +N_{5}(l ; q)-\frac{\phi(q)}{q}-\frac{3}{5} \frac{x^{(1 / 5)}}{q} \int_{1}^{\infty} \frac{\sum_{a \leq \mu} N_{5}\left(l a^{2} ; q\right)-((\phi(q)) / q)}{\mu^{(8 / 5)}} \mathrm{d} \mu \\
& +\frac{12}{25} \int_{1}^{\infty} \int_{1}^{\infty} \frac{\sum_{b \leq \lambda} \sum_{a \leq \mu} N_{5}\left(l a^{2} b ; q\right)-((\phi(q)) / q) \lambda \mu}{\mu^{(8 / 5)} \lambda^{(9 / 5)}} \mathrm{d} \lambda \mathrm{d} \mu .
\end{aligned}
$$

\section{Proof of Theorem 1}

First recall that any cube-full number can be written uniquely as $n=a^{3} b^{4} c^{5}$ where $b, c$ are square-free numbers and $(b, c)=1$. Following the proof of Theorem 2.3 in [20], we have

$$
\sum_{\substack{n \leq x, n \in \mathscr{P}_{3} \\ n \equiv l(\bmod q)}} 1=\sum_{\substack{a^{3} b^{4} c^{5} \leq x \\(b, c)=1, b, c \text { squarefree } \\ a^{3} b^{4} c^{5} \equiv l(\bmod q)}} 1=\sum_{\substack{a^{3} b^{4} c^{5} \leq x \\ a^{3} b^{4} c^{5} \equiv l(\bmod q) \\(b, c)=1}} \mu^{2}(b) \mu^{2}(c)
$$

In view of the identity $\mu^{2}(b)=\sum_{d^{2} \mid b} \mu(d)$, we get

$$
\sum_{\substack{n \leq x, n \in \mathscr{P}_{3} \\ n \equiv l(\bmod q)}} 1=\sum_{\substack{a^{3} b^{4} c^{5} d^{8} \leq x \\ a^{3} b^{4} c^{5} d^{8} \equiv l(\bmod q) \\(b d, c)=1}} \mu(d) \mu^{2}(c) .
$$

$$
\sum_{\substack{l|b \\ l| c}} \mu(l)= \begin{cases}1 & \text { if }(b, c)=1, \\ 0 & \text { otherwise, }\end{cases}
$$

and $\mu^{2}(c l)=\mu^{2}(c) \mu^{2}(l)$ for $(c, l)=1$, otherwise $\mu^{2}(c l)=0$ and $\mu^{2}(l) \mu(l)=\mu(l)$, we get

From the identity

$$
\sum_{\substack{n \leq x, n \in \mathscr{P}_{3} \\
n \equiv l(\bmod q)}} 1=\sum_{\substack{a^{3} b^{4} c^{5} d^{8} \leq x \\
a^{3} b^{4} c^{5} d^{8} \equiv l(\bmod q) \\
(c, d)=1}} \mu(d) \mu^{2}(c) \sum_{\begin{array}{c}
e \mid b \\
e \mid c
\end{array}} \mu(e)=\sum_{\begin{array}{c}
a^{3} b^{4} c^{5} d^{8} e^{9} \leq x \\
a^{3} b^{4} c^{5} d^{8} e^{9} \equiv l(\bmod q) \\
(c, e, d)=1
\end{array}} \mu(d) \mu^{2}(c) \mu(e) .
$$

Using again $\mu^{2}(c)=\sum_{f^{2} \mid c} \mu(f)$ and we have

$$
\sum_{\substack{g|c \\ g| e d}} \mu(g)= \begin{cases}1, & \text { if }(e d, c)=1, \\ 0, & \text { otherwise }\end{cases}
$$




$$
\begin{aligned}
& \sum_{\substack{n \leq x, n \in \mathscr{P}_{3} \\
n \equiv l(\bmod q)}} 1=\sum_{\begin{array}{c}
a^{3} b^{4} c^{5} d^{8} e^{9} \leq x \\
a^{3} b^{4} c^{5} d^{8} e^{9} \equiv l(\bmod q) \\
(c, e, d)=1
\end{array}} \mu(d) \mu(e) \sum_{f^{2} \mid c} \mu(f), \\
& =\sum_{\begin{array}{c}
a^{3} b^{4} c^{5} d^{8} e^{9} f^{10} \leq x \\
a^{3} b^{4} c^{5} d^{8} e^{9} f^{10} \equiv l(\bmod q) \\
(c f, e, d)=1
\end{array}} \mu(d) \mu(f) \mu(e) \\
& =\sum_{\begin{array}{c}
a^{3} b^{4} c^{5} d^{8} e^{9} f^{10} \leq x \\
a^{3} b^{4} c^{5} d^{8} e^{9} f^{10}=l(\bmod q) \\
(f, e, d)=1
\end{array}} \mu(d) \mu(f) \mu(e) \sum_{\substack{g|c \\
g| e d}} \mu(g) \\
& =\sum_{\begin{array}{c}
a^{3} b^{4} c^{5} g^{5} d^{8} e^{9} f^{10} \leq x \\
a^{3} b^{4} c^{5} g^{5} d^{8} e^{9} f^{10} \equiv l(\bmod q)
\end{array}} \mu(d) \mu(f) \mu(e) \sum_{g \mid e d} \mu(g) \\
& =\sum_{\substack{g^{5} d^{8} e^{9} f^{10} \leq x \\
(d, e, f)=1}} \mu(d) \mu(f) \mu(e) \sum_{g \mid e d} \mu(g) \sum_{\substack{a^{3} b^{4} c^{5} \leq\left(x / g^{5} d^{8} e^{9} f^{10}\right) \\
a^{3} b^{4} c^{5} \equiv l \\
g^{5} d^{8} e^{9} f^{10}(\bmod q)}} 1 .
\end{aligned}
$$

From $\left(x^{\eta} /\left(2^{J_{1}} q^{(1 / 2)}\right)\right) \ll 1$, we know that if $q \leq x^{2 \eta}$, then we can pick $2^{J_{1}}$ of size $\left(x^{\eta} / q^{(1 / 2)}\right)$, and if $q>x^{2 \eta}$, we simply pick $2^{J_{1}}=2$. Then, we obtain

$$
\begin{aligned}
\sum_{\substack{n \leq x, n \in \mathscr{P}_{3} \\
n \equiv l(\bmod q)}} 1=\sum_{\substack{g^{5} d^{8} e^{9} f^{10} \leq x q^{-(1 / 2 \eta)} \\
(d, e, f, q)=1}} \mu(d) \mu(f) \mu(e) \sum_{\substack{g \mid e d \\
(g, q)=1}} \mu(g) \sum_{\substack{a^{3} b^{4} c^{5} \leq\left(x / g^{5} d^{8} e^{9} f^{10}\right) \\
a^{3} b^{4} c^{5} \equiv l \overline{g^{5} d^{8} e^{9} f^{10}}(\bmod q)}} 1 \\
+\sum_{\substack{x q^{-(1 / 2 \eta)}<g^{5} d^{8} e^{9} f^{10} \leq x \\
(d, e, f, q)=1}} \mu(d) \mu(f) \mu(e) \sum_{\substack{g \mid e d \\
(g, q)=1}} \mu(g) \sum_{\substack{a^{3} b^{4} c^{5} \leq\left(x / g^{5} d^{8} e^{9} f^{10}\right) \\
a^{3} b^{4} c^{5} \equiv \overline{g^{5} d^{8} e^{9} f^{10}}(\bmod q)}} 1 .
\end{aligned}
$$

From the result of Theorem 2, we have

$$
\begin{aligned}
& \sum_{\substack{n \leqslant x, n \in \mathscr{P}_{3} \\
n \equiv l(\bmod q)}} 1=A_{q}^{\prime}(l) \sum_{\substack{d^{8} e^{9} f^{10} \geq 1 \\
(d, e, f, q)=1}} \frac{\mu(d) \mu(f) \mu(e)}{d^{(8 / 3)} e^{3} f^{(10 / 3)}} \sum_{\substack{g \mid e d \\
(g, q)=1}} \frac{\mu(g)}{g^{(5 / 3)}} \cdot \frac{x^{(1 / 3)}}{q} \\
& +B_{q}^{\prime}(l) \sum_{\substack{d^{8} e^{9} f^{10} \geq 1 \\
(d, e, f, q)=1}} \frac{\mu(d) \mu(f) \mu(e)}{d^{2} e^{(9 / 4)} f^{(5 / 2)}} \sum_{\substack{g \mid e d \\
(g, q)=1}} \frac{\mu(g)}{g^{(5 / 4)}} \cdot \frac{x^{(1 / 4)}}{q} \\
& +C_{q}^{\prime}(l) \sum_{\substack{d^{8} e^{9} f^{10} \geq 1 \\
(d, e, f, q)=1}} \frac{\mu(d) \mu(f) \mu(e)}{d^{(8 / 5)} e^{(9 / 5)} f^{2}} \sum_{\substack{g \mid e d \\
(g, q)=1}} \frac{\mu(g)}{g} \cdot \frac{x^{(1 / 5)}}{q} \\
& +O\left(\sum_{d^{8} e^{9} f^{10} \leqslant x q^{-(1 / 2 \eta)}} \frac{x^{(1 / 3)-(4 / 3) \eta}}{q^{(1 / 2)-\varepsilon}\left(d^{8} e^{9} f^{10}\right)^{(1 / 3)-(4 / 3) \eta}}+\sum_{d^{8} e^{9} f^{10} \leqslant x q^{-(1 / 2 \eta)}} \frac{x^{\eta+\lambda}}{\left(d^{8} e^{9} f^{10}\right)^{\eta+\lambda}} q^{\varepsilon}\right. \\
& \left.+\sum_{x q^{-(1 / 2 \eta)} \leq d^{8} e^{9} f^{10} \leq x} \frac{x^{(1 / 3)-(4 / 3) \eta}}{q^{(1 / 2)-\varepsilon}\left(d^{8} e^{9} f^{10}\right)^{(1 / 3)-(4 / 3) \eta}}+\sum_{x q^{-(1 / 2 \eta)} \leq d^{8} e^{9} f^{10} \leq x} \frac{x^{\lambda}}{\left(d^{8} e^{9} f^{10}\right)^{\lambda}} q^{(1 / 2)+\varepsilon}\right) \text {. }
\end{aligned}
$$

For the final term of the O-term, we have 


$$
\begin{aligned}
& \sum_{x q^{-(1 / 2)} \leq d^{8} e^{9} f^{10} \leq x} \frac{x^{\lambda}}{\left(d^{8} e^{9} f^{10}\right)^{\lambda}} q^{(1 / 2)+\varepsilon} \\
& \ll x^{\lambda} q^{(1 / 2)+\varepsilon} \sum_{d \leq x^{(1 / 8)}} \sum_{e \leq x^{(1 / 9)}} \sum_{\left.x q^{-(1 / 2)}\right)} \sum_{d^{-8} e^{-9} \leq f^{10} \leq \frac{x}{d^{8} e^{9}}} \frac{1}{d^{8 \lambda} e^{9 \lambda} f^{10 \lambda}} \\
& \ll x^{\lambda} q^{(1 / 2)+\varepsilon} \sum_{d \leq x^{(1 / 8)}} \frac{1}{d^{8 \lambda}} \sum_{e \leq x^{(1 / 9)}} \frac{1}{e^{9 \lambda}} \sum_{x q^{-(1 / 2))} d^{-8} e^{-9} \leq f^{10} \leq \frac{x}{d^{8} e^{9}}} \frac{1}{f^{10 \lambda}} \\
& \ll x^{\lambda} q^{(1 / 2)+\varepsilon} \sum_{d \leq x^{(1 / /)}} \frac{1}{d^{8 \lambda}} \sum_{e \leq x^{(1 / 9)}} \frac{1}{e^{9 \lambda}} \frac{x^{(1 / 10)-\lambda}}{d^{(8 / 10)-8 \lambda} e^{(9 / 10)-9 \lambda}} \\
& \ll x^{(1 / 10)} q^{(1 / 2)+\varepsilon} \sum_{d \leq x^{(1 / 8)}} \frac{1}{d^{(8 / 10)}} \sum_{e \leq x^{(1 / 9)}} \frac{1}{e^{(9 / 10)}} \\
& =x^{(49 / 360)} q^{(1 / 2)+\varepsilon} .
\end{aligned}
$$

In the same way, we have

$$
\sum_{x q^{-(1 / 2)} \leq d^{8} e^{9} f^{10} \leq x} \frac{x^{(1 / 3)-(4 / 3) \eta}}{q^{(1 / 2)-\varepsilon}\left(d^{8} e^{9} f^{10}\right)^{(1 / 3)-(4 / 3) \eta}} \ll x^{(49 / 360)} q^{\left(7 / 60 \mu_{1}\right)-(7 / 6)} \ll x^{(49 / 360)} q^{(1 / 2)+\varepsilon}
$$

The first two terms of the error term are

$$
\frac{x^{(1 / 3)-(4 / 3) \eta}}{q^{(1 / 2)-\varepsilon}}+x^{\eta+\lambda} q^{\varepsilon}
$$

If we take

$$
\frac{x^{(1 / 3)-(4 / 3) \eta}}{q^{(1 / 2)-\varepsilon}}=x^{\eta+\lambda} q^{\varepsilon}
$$

and assume $q=x^{\theta}$, then we have $\eta=(1 / 7)-(3 / 7) \lambda-(3 / 14) \theta$ and

$$
x^{\eta+\lambda} q^{\varepsilon}=\frac{x^{(1 / 7)+(4 / 7) \lambda}}{q^{(3 / 14)} q^{\varepsilon}} .
$$

Now we pick $\lambda=(5 / 51)+\varepsilon$ and get

$$
\frac{x^{(1 / 3)-(4 / 3) \eta}}{q^{(1 / 2)-\varepsilon}}+x^{\eta+\lambda} q^{\varepsilon} \ll \frac{x^{(71 / 357)}}{q^{(3 / 14)}} q^{\varepsilon} .
$$

Consequently, we obtain

$$
\begin{aligned}
\sum_{\substack{n \leq x, n \in \mathscr{P}_{3} \\
n=l(\bmod q)}} 1= & A_{q}^{\prime}(l) \sum_{\substack{d^{8} e^{9} f^{10} \geq 1 \\
(d, e, f, q)=1}} \frac{\mu(d) \mu(f) \mu(e)}{d^{(8 / 3)} e^{3} f^{(10 / 3)}} \sum_{\substack{g \mid e d \\
(g, q)=1}} \frac{\mu(g)}{g^{(5 / 3)}} \cdot \frac{x^{(1 / 3)}}{q} \\
& +B_{q}^{\prime}(l) \sum_{\substack{d^{8} e^{9} f^{10} \geq 1 \\
(d, e, f, q)=1}} \frac{\mu(d) \mu(f) \mu(e)}{d^{2} e^{(9 / 4)} f^{(5 / 2)}} \sum_{\substack{g \mid e d \\
(g, q)=1}} \frac{\mu(g)}{g^{(5 / 4)}} \cdot \frac{x^{(1 / 4)}}{q} \\
& +C_{q}^{\prime}(l) \sum_{\substack{d^{8} e^{9} f^{10} \geq 1 \\
(d, e, f, q)=1}} \frac{\mu(d) \mu(f) \mu(e)}{d^{(8 / 5)} e^{(9 / 5)} f^{2}} \sum_{\substack{g \mid e d \\
(g, q)=1}} \frac{\mu(g)}{g} \cdot \frac{x^{(1 / 5)}}{q}+O\left(\left(x^{(49 / 360)} q^{(1 / 2)}+\frac{x^{(71 / 357)}}{q^{(3 / 14)}}\right) q^{\varepsilon}\right),
\end{aligned}
$$

which is Theorem 2 . 


\section{Data Availability}

The data used to support the findings of this study are included within the article.

\section{Conflicts of Interest}

The authors declare that they have no conflicts of interest.

\section{Acknowledgments}

This research was funded by the National Natural Science Foundation of China (nos. 11871317, 11926325, and 11926321).

\section{References}

[1] P. Erdös, G. Szekeres, ÜBer die Anzahl der Abelschen Gruppen gegehener Ordnung und über ein verwandtes zahlentheoretisches Problem," Acta Scientiarum Mathematicarum, vol. 7, pp. 95-102, 1934.

[2] R. Balasubramanian, K. Ramachandra, and M. Subbarao, "On the error function in the asymptotic formula for the counting function of k-full numbers," Acta Arithmetica, vol. 50, no. 2, pp. 107-118, 1988.

[3] P. T. Bateman and E. Grosswald, "On a theorem of Erdös and Szekeres," Illinois Journal of Mathematics, vol. 2, no. 1, pp. 88-98, 1958.

[4] Y. C. Cai, "On the distribution of square-full integers," Acta Mathematica Sinica (N.S.), vol. 13, pp. 269-280, 1997.

[5] X. D. Cao, "On the distribution of square-full integers," Periodica Mathematica Hungarica, vol. 34, pp. 169-175, 1997.

[6] S. W. Golomb, "Powerful numbers," The American Mathematical Monthly, vol. 77, no. 8, pp. 848-855, 1970.

[7] A. Ivić, "On the asymptotic formulas for powerful numbers," Publications de l'Institut Mathématique, vol. 23, no. 37, pp. 85-94, 1978.

[8] A. Ivić, The Riemann Zeta-Function: Theory and Applications, Dover Publications, Inc., Mineola, NY, USA, 2003.

[9] A. Ivić and P. Shiu, "The distribution of the powerful numbers," International Journal of Mathematics, vol. 26, pp. 576-590, 1982.

[10] E. Krätzel, "On the distribution of square-full and cube-full numbers," Monatshefte für Mathematik, vol. 120, no. 2, pp. 105-119, 1995.

[11] H. Q. Liu, "The distribution of square-full integers," Archiv der Mathematik, vol. 32, no. 2, pp. 449-454, 1994.

[12] H. Q. Liu, "On the distribution of square-full numbers," Journal of Harbin Institute of Technology, vol. 5, pp. 4-6, 1998.

[13] M. Munsch, I. E. Shparlinski, and K. H. Yau, "Smooth squarefree and squarefull integers in arithmetic progressions," Mathematika, vol. 66, no. 1, pp. 56-70, 2020.

[14] W. G. Nowak and M. Schmeier, "Conditional asymptotic formula for a class of arithmetic functions," American Mathematical Society, vol. 103, pp. 712-717, 1988.

[15] D. Suryanarayana and R. Sita Rama Chandra Rao, "The distribution of square-full integers," Arkiv För Matematik, vol. 11, no. 1-2, pp. 195-201, 1973.

[16] J. Wu, "On the distribution of square-full and cube-full integers," Monatshefte För Mathematik, vol. 126, no. 4, pp. 353-367, 1998.

[17] J. Wu, "On the distribution of square-full integers," Archiv der Mathematik, vol. 77, no. 3, pp. 233-240, 2001.
[18] W. Y. Zhu and K. T. Yu, "The distribution of square-full integers," Pure Appl. Math. (Xi'an), vol. 12, no. 2, pp. 113-122, 1996.

[19] H. Liu and T. Zhang, "On the distribution of square-full numbers in arithmetic progressions," Archiv der Mathematik, vol. 101, no. 1, pp. 53-64, 2013.

[20] T. Srichan, "Square-full and cube-full numbers in arithmetic progressions," Šiauliai Mathematical Seminar, vol. 8, no. 16, pp. 223-248, 2013.

[21] T. H. Chan, "Squarefull numbers in arithmetic progression II," Journal of Number Theory, vol. 152, pp. 90-104, 2015.

[22] T. H. Chan and K. M. Tsang, "Squarefull numbers in arithmetic progressions," International Journal of Number Theory, vol. 9, no. 4, pp. 885-901, 2013.

[23] H. L. Montgomery and R. C. Vaughan, "Exponential sums with multiplicative coefficients," Inventiones Mathematicae, vol. 43, no. 1, pp. 69-82, 1977.

[24] H. Iwaniec and E. Kowalski, "Analytic number theory," American Mathematical Society, vol. 53, 2004.

[25] K. Liu, I. E. Shparlinski, and T. Zhang, "Average distribution of k-free numbers in arithmetic progressions," Mathematische Nachrichten, vol. 293, no. 8, pp. 1505-1514, 2020.

[26] C. J. Moreno and O. Moreno, "Exponential sums and Goppa codes. I," Proceedings of the American Mathematical Society, vol. 111, no. 2, p. 523, 1991.

[27] E. Bombieri, "On exponential sums in finite fields," American Journal of Mathematics, vol. 88, no. 1, pp. 71-105, 1966.

[28] T. M. Apostol, Introduction to Analytic Number Theory, Springer-Verlag, Heidelberg, NY, USA, 1976. 